\title{
DONALDSON-THOMAS INVARIANTS OF LOCAL ELLIPTIC SURFACES VIA THE TOPOLOGICAL VERTEX
}

\author{
JIM BRYAN ${ }^{1}$ and MARTIJN KOOL ${ }^{2}$ \\ ${ }^{1}$ Department of Mathematics, University of British Columbia, Room 121, \\ 1984 Mathematics Road, Vancouver, B.C., Canada V6T 1 Z2 \\ ${ }^{2}$ Mathematical Institute, Utrecht University, Room 502, Budapestlaan 6, \\ 3584 CD Utrecht, The Netherlands
}

Received 7 January 2019; accepted 28 January 2019

\begin{abstract}
We compute the Donaldson-Thomas invariants of a local elliptic surface with section. We introduce a new computational technique which is a mixture of motivic and toric methods. This allows us to write the partition function for the invariants in terms of the topological vertex. Utilizing identities for the topological vertex proved in Bryan et al. ['Trace identities for the topological vertex', Selecta Math. (N.S.) 24 (2) (2018), 1527-1548, arXiv:math/1603.05271], we derive product formulas for the partition functions. The connected version of the partition function is written in terms of Jacobi forms. In the special case where the elliptic surface is a K3 surface, we get a derivation of the Katz-Klemm-Vafa formula for primitive curve classes which is independent of the computation of Kawai-Yoshioka.
\end{abstract}

2010 Mathematics Subject Classification: 14N35, 14C05

\section{Introduction}

Let $p: S \rightarrow B$ be a nontrivial elliptic surface over a complex smooth projective curve $B$. We assume $p$ has a section and all singular fibers are irreducible rational nodal curves.

In this paper, we study the Donaldson-Thomas (DT) invariants of $X=\operatorname{Tot}\left(K_{S}\right)$, that is, the total space of the canonical bundle $K_{S}$. This is a noncompact CalabiYau threefold. Let $\beta$ be an effective curve class on $S$. Consider the Hilbert scheme

$$
\operatorname{Hilb}^{\beta, n}(X)=\left\{Z \subset X:[Z]=\beta, \chi\left(\mathcal{O}_{Z}\right)=n\right\}
$$

(c) The Author(s) 2019. This is an Open Access article, distributed under the terms of the Creative Commons Attribution licence (http://creativecommons.org/licenses/by/4.0/), which permits unrestricted re-use, distribution, and reproduction in any medium, provided the original work is properly cited. 
of proper subschemes $Z \subset X$ with homology class $\beta$ and holomorphic Euler characteristics $n$. The DT invariants of $X$ can be defined as

$$
\mathrm{DT}_{\beta, n}(X):=e\left(\operatorname{Hilb}^{\beta, n}(X), v\right):=\sum_{k \in \mathbb{Z}} k e\left(v^{-1}(k)\right),
$$

where $e(\cdot)$ denotes topological Euler characteristic and $v: \operatorname{Hilb}^{\beta, n}(X) \rightarrow \mathbb{Z}$ is Behrend's constructible function [2]. We also consider an unweighted Euler characteristic version of these invariants

$$
\widehat{\mathrm{DT}}_{\beta, n}(X):=e\left(\operatorname{Hilb}^{\beta, n}(X)\right) \text {. }
$$

We choose a section $B \subset S$ and focus on the primitive classes $\beta=B+d F$, where $B$ is the class of the chosen section and $F$ the class of the fiber. We define the partition functions by

$$
\begin{aligned}
& \widehat{\mathrm{DT}}(X)=\sum_{d=0}^{\infty} \sum_{n \in \mathbb{Z}} \widehat{\mathrm{DT}}_{B+d F, n}(X) p^{n} q^{d}, \\
& \mathrm{DT}(X)=\sum_{d=0}^{\infty} \sum_{n \in \mathbb{Z}} \mathrm{DT}_{B+d F, n}(X) y^{n} q^{d} .
\end{aligned}
$$

We also consider the partition functions for the invariants for multiples of the fiber class

$$
\begin{aligned}
& \widehat{\mathrm{DT}}_{\text {fib }}(X)=\sum_{d=0}^{\infty} \sum_{n \in \mathbb{Z}} \widehat{\mathrm{DT}}_{d F, n}(X) p^{n} q^{d}, \\
& \mathrm{DT}_{\text {fib }}(X)=\sum_{d=0}^{\infty} \sum_{n \in \mathbb{Z}} \mathrm{DT}_{d F, n}(X) y^{n} q^{d} .
\end{aligned}
$$

The main results of this paper are closed product formulas for the partition functions $\widehat{\mathrm{DT}}(X)$ and $\widehat{\mathrm{DT}}_{\text {fib }}(X)$. Assuming a general conjecture about the Behrend function, we also determine $\mathrm{DT}(X)$ and $\mathrm{DT}_{\text {fib }}(X)$.

We use the notation

$$
M(p, q)=\prod_{m=1}^{\infty}\left(1-p^{m} q\right)^{-m}
$$

and the shorthand $M(p)=M(p, 1)$. 
THEOREM 1. Let $e(S)$ and $e(B)$ denote the topological Euler characteristics of the elliptic surface and the base. Then

$$
\begin{aligned}
\widehat{\mathrm{DT}}(X)= & \left\{M(p) \prod_{d=1}^{\infty} \frac{M\left(p, q^{d}\right)}{\left(1-q^{d}\right)}\right\}^{e(S)} \\
& \times\left\{\frac{1}{\left(p^{1 / 2}-p^{-1 / 2}\right)} \prod_{d=1}^{\infty} \frac{\left(1-q^{d}\right)}{\left(1-p q^{d}\right)\left(1-p^{-1} q^{d}\right)}\right\}^{e(B)} \\
\widehat{\mathrm{DT}}_{\mathrm{fib}}(X)= & \left\{M(p) \prod_{d=1}^{\infty} M\left(p, q^{d}\right)\right\}^{e(S)}\left\{\prod_{d=1}^{\infty} \frac{1}{\left(1-q^{d}\right)}\right\}^{e(B)} .
\end{aligned}
$$

The formula for $\widehat{\mathrm{DT}}_{\text {fib }}(X)$ was previously proved using wall-crossing methods by Toda. (After applying the Pandharipande-Thomas/Donaldson-Thomas (PT/DT) correspondence [4], this is essentially [18, Theorem 6.9].)

The ratio $\widehat{\mathrm{DT}}(X) / \widehat{\mathrm{DT}}_{\text {fib }}(X)$ can be considered as the generating function for the connected invariants in the classes $B+d F$. This series has a particularly nice form and can be written in terms of classical Jacobi forms. Consider the Dedekind eta function and the Jacobi theta function

$$
\begin{aligned}
\eta & =q^{1 / 24} \prod_{k=1}^{\infty}\left(1-q^{k}\right), \\
\Theta & =\left(p^{1 / 2}-p^{-1 / 2}\right) \prod_{k=1}^{\infty} \frac{\left(1-p q^{k}\right)\left(1-p^{-1} q^{k}\right)}{\left(1-q^{k}\right)^{2}} .
\end{aligned}
$$

COROLLARY 2. The partition function of the connected invariants is given as follows

$$
\frac{\widehat{\mathrm{DT}}(X)}{\widehat{\mathrm{DT}}_{\text {fib }}(X)}=\left(q^{-1 / 24} \eta\right)^{-e(S)} \Theta^{-e(B)} .
$$

In the case where $S \rightarrow \mathbb{P}^{1}$ is an elliptically fibered K3 surface, the above series specializes (up to a factor of $q$ ) to the reciprocal of $\eta^{24} \Theta^{2}$, the unique Jacobi cusp form of weight 10 and index 1 . This is the Jacobi form appearing in the wellknown Katz-Klemm-Vafa (KKV) formula. In order to obtain the KKV formula, we require the connected series, because $X$ is noncompact.

Our result provides a new derivation of the KKV formula for primitive classes. (At least for the Euler characteristic version of the DT invariants. For the Behrend function weighted DT invariants, we require Conjecture 21, see Theorem 3.) The $\mathrm{KKV}$ formula was proved in all curve classes in [17]. The appearance of the 
Jacobi form $\eta^{24} \Theta^{2}$ in previous proofs of the KKV formula [14, 17] ultimately comes from the calculation of Euler characteristics of relative Hilbert schemes of points on curves on K3 by Kawai-Yoshioka [10]. Our derivation of the KKV formula is the first that does not depend on the Kawai-Yoshioka formula.

Our results can be extended to apply to the usual (Behrend function weighted) DT invariants if we assume a general conjecture that we formulate in Section 8. Our conjecture relates the Behrend function at subschemes with embedded points to the value of the Behrend function at the underlying Cohen-Macaulay subscheme and may be of independent interest.

Theorem 3. Assume that Conjecture 21 holds, then

$$
\operatorname{DT}(X)=(-1)^{x\left(\mathcal{O}_{S}\right)} \widehat{\mathrm{DT}}(X)
$$

and

$$
\mathrm{DT}_{\mathrm{fib}}(X)=\widehat{\mathrm{DT}}_{\mathrm{fib}}(X)
$$

under the change of variables

$$
y=-p .
$$

A similar phenomenon to the above is known to hold when $X$ is a toric CalabiYau threefold.

The method of computation that we introduce in this paper has been applied to other elliptically fibered geometries. Indeed, it has found applications to the calculation of DT generating functions on $\mathrm{K} 3 \times E$, where $E$ is an elliptic curve [5] and abelian threefolds [7], and is expected to apply to $(\mathrm{K} 3 \times E) / G$ where $G$ is a finite group acting symplectically on each factor.

Although the geometry under consideration is not toric, we combine $\mathbb{C}^{*}$ localization, motivic methods, and $\left(\mathbb{C}^{*}\right)^{3}$-localization to end up with expressions that only depend on the topological vertex $\mathrm{V}_{\lambda \mu \nu}$, and the topological Euler characteristics $e(B), e(S)$. The outline of our method is as follows:

- The $\mathbb{C}^{*}$-action on $X$ induces an action on $\operatorname{Hilb}(X)$ whose Euler characteristic localizes to the $\mathbb{C}^{*}$-fixed locus. In Section 3 we show that any $\mathbb{C}^{*}$-invariant subscheme has a maximal Cohen-Macaulay subscheme which is a curve of a special form which we call a partition thickened comb curve (Definition 7). This curve is determined by data consisting of points $x_{i} \in B$ labeled by integer partitions $\lambda^{(i)}$. This gives rise to a constructible morphism $\rho$ to $\operatorname{Sym} B$ taking the value $\sum_{i}\left|\lambda^{(i)}\right| x_{i}$ on such a curve (see Theorem 8 ).

- In Section 4, we push forward the Euler characteristic measure to Sym $B$ via the map $\rho$. We show that $\rho_{*}(1)$, the push-forward measure, has nice multiplicative 
properties that allow us to compute the weighted Euler characteristic over Sym $B$ using a general result about symmetric products (Lemma 32).

- To compute the push-forward measure $\rho_{*}(1)$ explicitly, we must compute the Euler characteristics of the fibers of $\rho$. These fibers are strata in the Hilbert scheme parameterizing subschemes whose maximal Cohen-Macaulay subscheme is a fixed partition thickened comb curve $C$. Here it is useful to switch from Hilbert schemes with fixed $C$ to Quot schemes of its ideal sheaf $I_{C}$. We introduce a further stratification of these Quot schemes by specifying the set-theoretic support of the quotients. This allows us to write these Quot schemes as products (in $K$-theory) of Quot schemes of $I_{C}$ where the quotient is supported only on one of the nodes of $C_{\text {red }}$, or only on one of the components of $C_{\text {red }}$ (minus the nodes), or only on the complement of $C$. The Quot scheme of quotients supported at a node of $C_{\text {red }}$ can be expressed (in $K$-theory) as a Quot scheme of a partition thickened comb curve (determined by $C$ ) on $\mathbb{C}^{3}$. Similarly, after further push-forwards to further symmetric products, we express the Euler characteristics of all other Quot schemes in terms of Euler characteristics of Quot schemes on $\mathbb{C}^{3}$ as well (see Section 5).

- The Quot schemes of $\mathbb{C}^{3}$ of the previous step all carry a natural $T=\left(\mathbb{C}^{*}\right)^{3}$ action. $T$-localization then allows us to write their Euler characteristics in terms of the topological vertex (see Section 6).

- Finally, using the trace formulas for the topological vertex proved in [6], we write our expression for $\widehat{\mathrm{DT}}(X)$ as the closed product formula given in Theorem 1.

Our proof of Theorem 3 requires Theorem 24, an involved computation of $\operatorname{Ext}_{0}^{1}\left(I_{C}, I_{C}\right)$ for partition thickened comb curves $C$. The proof of Theorem 24 occupies most of Section 9 and while technical in nature, the method we introduce (again a mixture of formally local toric methods and global geometry) may be of independent interest to the experts.

\section{Definitions, notation, and conventions}

Let $p: S \rightarrow B$ be an elliptic surface over a smooth projective curve $B$. We assume:

(1) $S$ is a nontrivial fibration;

(2) $p$ has a section $B \subset S$;

(3) all singular fibers of $p$ are irreducible rational nodal curves. 
We note that the number of singular fibers is equal to $e(S)$.

We write $F_{x}$ for the fiber $p^{-1}(x)$ over a point $x \in B$. We choose a section $B \subset S$ and denote its class in $H_{2}(S)$ by $B$ as well. We denote the class of the fiber by $F \in H_{2}(S)$.

Let $X=\operatorname{Tot}\left(K_{S}\right)$ be the total space of the canonical bundle $K_{S}$. For brevity, we define

$$
\begin{aligned}
\operatorname{Hilb}^{d, n}(X) & :=\operatorname{Hilb}^{B+d F, n}(X), \\
\widehat{\mathrm{DT}}_{d, n}(X) & :=\widehat{\mathrm{DT}}_{B+d F, n}(X) .
\end{aligned}
$$

Since we are dealing with generating functions and our calculations involve motivic methods on the Hilbert schemes, it is useful to introduce the following notation. We define

$$
\operatorname{Hilb}^{d, \bullet}(X):=\sum_{n \in \mathbb{Z}} \operatorname{Hilb}^{d, n}(X) p^{n},
$$

where we view the right hand side (RHS) as a formal Laurent series whose coefficients are elements in the Grothendieck ring of varieties, that is, $K_{0}\left(\operatorname{Var}_{\mathbb{C}}\right)((p))$.

Convention 3.1. When an index is replaced by a bullet, we will multiply by the appropriate variable and sum over the index. We regard the result as a formal power (or Laurent) series whose coefficients lie in $K_{0}\left(\operatorname{Var}_{\mathbb{C}}\right)$ and we extend operations of the Grothendieck group (addition, multiplication, Euler characteristic) to the series in the obvious way.

For example

$$
\operatorname{Hilb}^{\bullet \bullet \bullet}(X)=\sum_{d=0}^{\infty} \sum_{n \in \mathbb{Z}} \operatorname{Hilb}^{d, n}(X) q^{d} p^{n} \in K_{0}\left(\operatorname{Var}_{\mathbb{C}}\right)((p))[[q]],
$$

so that we can write

$$
\widehat{\mathrm{DT}}(X)=e\left(\operatorname{Hilb}^{\bullet \bullet \bullet}(X)\right) \text {. }
$$

It is notationally convenient to treat an Euler characteristic weighted by a constructible function as a Lebesgue integral, where the measurable sets are constructible sets, the measurable functions are constructible functions, and the measure of a set is given by its Euler characteristic. In this language we have

$$
\widehat{\mathrm{DT}}_{d, n}(X)=\int_{\operatorname{Hilb}^{d, n}(X)} 1 d e, \quad \mathrm{DT}_{d, n}(X)=\int_{\operatorname{Hilb}^{d, n}(X)} v d e
$$


and following the bullet convention we have

$$
\widehat{\mathrm{DT}}(X)=\int_{\mathrm{Hilb}^{\bullet \bullet}(X)} 1 d e, \quad \mathrm{DT}(X)=\int_{\mathrm{Hilb}^{\bullet \bullet}(X)} v d e .
$$

We will also need notation for subsets of the Hilbert scheme which parameterize those subschemes obtained by adding embedded points and/or zero-dimensional components to some fixed Cohen-Macaulay curve.

Definition 4. Let $C \subset X$ be a (not necessarily reduced) Cohen-Macaulay subscheme of dimension 1. Consider the Hilbert scheme of subschemes $Z \subset X$ of class $[Z]=[C] \in H_{2}(X)$ and $\chi\left(\mathcal{O}_{Z}\right)=\chi\left(\mathcal{O}_{C}\right)+n$. Inside this Hilbert scheme, we define the following closed subset

$\operatorname{Hilb}^{n}(X, C)=\left\{Z \subset X\right.$ such that $C \subset Z$ and $I_{C} / I_{Z}$ has finite length $\left.n\right\}$.

Once the Cohen-Macaulay curve $C \subset X$ is fixed, it is useful to work with the Quot scheme Quot ${ }_{X}^{n}\left(I_{C}\right)$ of zero-dimensional quotients of $I_{C}$ of length $n$. We have the following lemma.

LEMMA 5. The following equality holds in $K_{0}\left(\operatorname{Var}_{\mathbb{C}}\right)((p))$

$$
\operatorname{Hilb}^{\bullet}(X, C)=\operatorname{Quot}_{X}^{\bullet}\left(I_{C}\right) .
$$

Proof. The universal quotient $I_{C \times \operatorname{Quot}_{X}^{n}\left(I_{C}\right)} \rightarrow Q$ has flat kernel $I_{\mathcal{Z}}$. This provides a flat family $\mathcal{Z} \subset X \times \operatorname{Quot}_{X}^{n}\left(I_{C}\right)$ which gives a morphism to the Hilbert scheme. The kernel of a quotient $I_{C} \rightarrow Q$, where $Q$ is zero-dimensional of length $n$, is an ideal sheaf $I_{Z} \subset I_{C}$ satisfying

$$
n=\chi(Q)=\chi\left(I_{C} / I_{Z}\right)=\chi\left(\mathcal{O}_{Z}\right)-\chi\left(\mathcal{O}_{C}\right) .
$$

Every $\mathbb{C}$-valued point of $\operatorname{Hilb}^{n}(X, C)$ arises from a quotient $I_{Z} \rightarrow Q$ in this way. This gives a geometric bijection $\operatorname{Quot}_{X}^{n}\left(I_{C}\right) \rightarrow \operatorname{Hilb}^{n}(X, C)$ from which the lemma follows.

\section{Reduction to partition thickened comb curves}

The action of $\mathbb{C}^{*}$ on the fibers of $X$ lifts to the moduli space $\operatorname{Hilb}^{d, \bullet}(X)$. Therefore

$$
\left.\int_{\operatorname{Hilb}^{d} \cdot(X)} 1 d e=\int_{\operatorname{Hilb}^{d} \cdot \bullet} 1 X\right)^{\mathbb{C}^{*}}
$$


The main result of this section is a classification of the subschemes parameterized by $\operatorname{Hilb}^{d, n}(X)^{\mathbb{C}^{*}}$, namely the $\mathbb{C}^{*}$-invariant subschemes. We find that the maximal Cohen-Macaulay subscheme of a $\mathbb{C}^{*}$-invariant subscheme is determined by a point in $\mathrm{Sym}^{d}(B)$ along with some discrete data (a collection of integer partitions). We begin with some notation.

Definition 6. Let $T=\operatorname{Tot}\left(\left.K_{S}\right|_{B}\right)$ and let $p: X \rightarrow T$ be the elliptic fibration induced by the elliptic fibration $p: S \rightarrow B$. We say that a subscheme $C \subset X$ is a comb curve if $C=B \cup p^{-1}(Z)$ where $Z \subset T$ is a zero-dimensional subscheme which is set-theoretically supported on $B$.

Let $\lambda=\left(\lambda_{1} \geqslant \cdots \geqslant \lambda_{l}\right)$ be an integer partition. Then $\lambda$ determines a zerodimensional subscheme $Z_{\lambda} \subset \operatorname{Spec} \mathbb{C}[[r, s]]$ given by the monomial ideal

$$
I_{\lambda}=\left(r^{\lambda_{1}}, r^{\lambda_{2}} s, \ldots, r^{\lambda_{l}} s^{l-1}, s^{l}\right) .
$$

In terms of $\lambda$ as a Young diagram, we note $(\rho, \sigma) \in \lambda$ if and only if $r^{\rho} s^{\sigma} \notin I_{\lambda}$.

Definition 7. Let $C=B \cup p^{-1}(Z)$ be a comb curve, let $x_{1}, \ldots, x_{n} \in B \subset T$ be the points where $Z$ is supported, and let $\left(r_{i}, s_{i}\right)$ be formal local coordinates on $T$ about each point $x_{i}$ so that $s_{i}$ vanishes on $S \cap T$ and $r_{i}$ vanishes on $R_{i} \cap T$ where $R_{i}=\operatorname{Tot}\left(\left.K_{S}\right|_{F_{x_{i}}}\right)$. We say that $C$ is a partition thickened comb curve if there exist partitions $\lambda^{(1)}, \ldots, \lambda^{(n)}$ such that $Z$ is given by $Z_{\lambda^{(i)}}$ in the local coordinates $\left(r_{i}, s_{i}\right)$ about $x_{i}$. We denote such a curve by $B \cup_{i}\left(\lambda^{(i)} F_{x_{i}}\right)$. (Specifically, writing $r:=r_{i}, s:=s_{i}, \lambda=\left(\lambda_{1} \geqslant \cdots \geqslant \lambda_{l}\right):=\lambda^{(i)}, x:=x_{i}, F:=F_{x_{i}}$, and $t$ for a coordinate at $x$ vanishing on $T$, the ideal of $B \cup \lambda F$ in a formal neighborhood of $x$ is given by $(s, t) \cdot\left(r^{\lambda_{1}}, r^{\lambda_{2}} s, \ldots, r^{\lambda_{l}} s^{l-1}, s^{l}\right) \subset \mathbb{C}[[r, s, t]]$.) We say that a subscheme $Z \subset X$ is a partition thickened comb curve with points (PCP) if the maximal Cohen-Macaulay subscheme $Z_{\mathrm{CM}} \subset Z$ is a partition thickened comb curve, in other words, $Z$ is obtained from a partition thickened comb curve by adding embedded points and/or zero-dimensional components. We denote by

$$
\operatorname{Hilb}_{\mathrm{PCP}}^{d, n}(X) \subset \operatorname{Hilb}^{d, n}(X)
$$

the locus in the Hilbert scheme parameterizing PCP.

In the next section it will be important to notationally distinguish between singular and smooth fibers. See Figure 1 for an illustration of a partition thickened comb curve with smooth fibers $\left\{F_{x_{i}}\right\}$ thickened by partitions $\left\{\lambda^{(i)}\right\}$ and nodal fibers $\left\{F_{y_{j}}\right\}$ thickened by partitions $\left\{\mu^{(j)}\right\}$.

Crucially, any effective divisor on $S$ in class $[B+d F]$ is a comb curve, that is, the scheme-theoretic union of $B$ (our chosen section) and some (possibly 


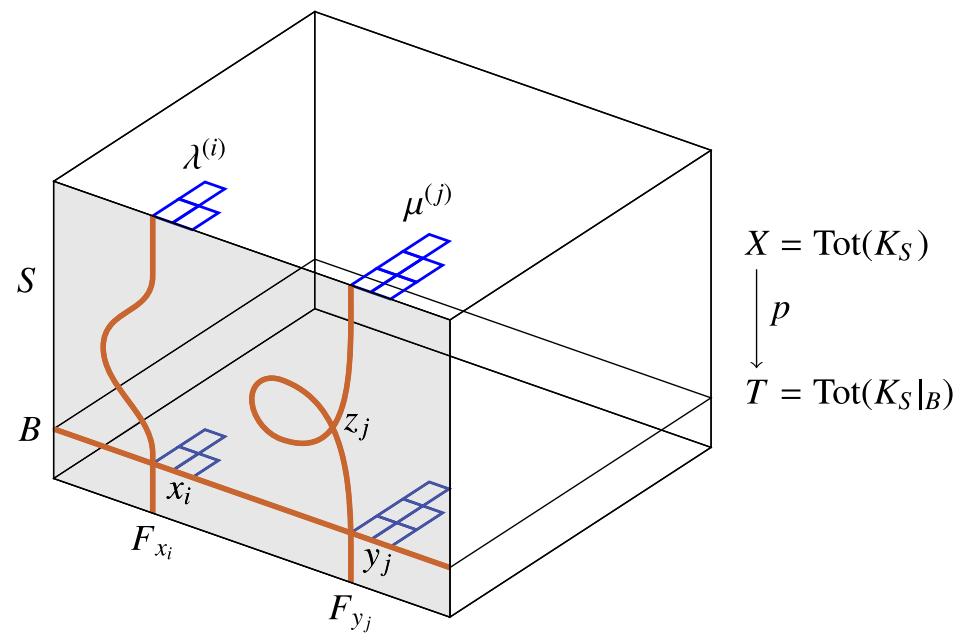

Figure 1. A partition thickened comb curve $C=B \cup_{i}\left(\lambda^{(i)} F_{x_{i}}\right) \cup_{j}\left(\mu^{(j)} F_{y_{j}}\right)$.

thickened) fibers with total multiplicity $d$. This is proved in Lemma 31. The main result of this section is the following:

THEOREM 8. If a subscheme $Z \subset X$ in the class $[Z]=B+d F$ is $\mathbb{C}^{*}$-invariant, then it is a PCP. That is

$$
\operatorname{Hilb}^{d, n}(X)^{\mathbb{C}^{*}} \subset \operatorname{Hilb}_{\mathrm{PCP}}^{d, n}(X) \subset \operatorname{Hilb}^{d, n}(X) .
$$

Moreover, $\mathbb{C}^{*}$ acts on $\mathrm{Hilb}_{\mathrm{PCP}}^{d, n}(X)$ and there exists a constructible morphism

$$
\rho_{d}: \operatorname{Hilb}_{\mathrm{PCP}}^{d, \bullet}(X) \rightarrow \operatorname{Sym}^{d}(B)
$$

such that if $[Z] \in \operatorname{Hilb}_{\mathrm{PCP}}^{d, n}(X)$, where the maximal Cohen-Macaulay subscheme of $Z$ is $B \cup_{i}\left(\lambda^{(i)} F_{x_{i}}\right)$, then

$$
\rho_{d}([Z])=\sum_{i}\left|\lambda^{(i)}\right| x_{i}
$$

Proof. We have to prove the following: Let $Z \subset X$ be a $\mathbb{C}^{*}$-fixed subscheme in the class $[Z]=B+d F$, then the underlying Cohen-Macaulay support curve $C$ is a partition thickened comb curve. Let $I_{C} \subset \mathcal{O}_{X}$ be the ideal sheaf 
defining $C$. Pushing forward along the projection $\pi: X=K_{S} \rightarrow S$ and using the decomposition into $\mathbb{C}^{*}$-weight spaces shows that there exist ideal sheaves

$$
I_{0} \subset \cdots \subset I_{l-1} \subsetneq \mathcal{O}_{S}
$$

such that

$$
\pi_{*} I_{C}=\bigoplus_{i=0}^{l-1} I_{i} \otimes K_{S}^{-i} .
$$

This is essentially proved in [11, Section 4] (albeit in the PT rather than the DT setting). Each $I_{i}$ defines a closed subscheme $C_{i} \subset S$ satisfying

$$
\begin{gathered}
S \supsetneq C_{0} \supset \cdots \supset C_{l-1}, \\
\sum_{i=0}^{l-1}\left[C_{i}\right]=B+d F \in H_{2}(S) .
\end{gathered}
$$

Therefore each $C_{i}$ has dimension $\leqslant 1$. In fact each $C_{i} \subset S$ is a Cohen-Macaulay curve, or else $C$ has embedded points. Since a Cohen-Macaulay curve on a surface is Gorenstein, each $C_{i}$ is an effective divisor.

By the nesting condition and Lemma 31, we deduce

$$
\begin{gathered}
C_{0}=B+\sum_{i=1}^{n} \lambda_{1}^{(i)} F_{x_{i}}, \\
C_{1}=\sum_{i=1}^{n} \lambda_{2}^{(i)} F_{x_{i}}, \\
\vdots \\
C_{l-1}=\sum_{i=1}^{n} \lambda_{l}^{(i)} F_{x_{i}},
\end{gathered}
$$

for some distinct points $x_{1}, \ldots, x_{n} \in B$ and $\lambda_{1}^{(i)} \geqslant \cdots \geqslant \lambda_{l}^{(i)}$. This proves that the $\mathbb{C}^{*}$-fixed locus lies inside the PCP locus.

Since the $\mathbb{C}^{*}$-invariant Cohen-Macaulay curves just described are exactly the support curves of PCP curves, it follows that the PCP locus is $\mathbb{C}^{*}$-invariant. Finally, since the assignment $Z \mapsto Z_{\mathrm{CM}}$ which takes a 1-dimensional subscheme to its maximal Cohen-Macaulay subscheme defines a constructible morphism $\operatorname{Hilb}(X) \rightarrow \operatorname{Hilb}(X)$, its restriction to $\operatorname{Hilb}_{\mathrm{PCP}}^{d, \bullet}(X)$ is also constructible and thus gives the constructible morphism $\rho_{d}$. 


\section{Push-forward to the symmetric product}

From the $\mathbb{C}^{*}$-equivariant inclusions in Theorem 8 and $\mathbb{C}^{*}$-localization of Euler characteristic, we have

$$
\widehat{\mathrm{DT}}(X)=\int_{\mathrm{Hilb}^{\bullet \bullet}(X)} 1 d e=\int_{\mathrm{Hilb}^{\bullet \bullet \bullet}(X)^{\mathrm{C}^{*}}} 1 d e=\int_{\operatorname{Hilb}_{\mathrm{PCP}} \cdot{ }^{\circ}(X)} 1 d e .
$$

We compute these Euler characteristics by pushing forward along the map $\rho_{d}$ constructed in Theorem 8 . That is we use

$$
\int_{\mathrm{Hilb}_{\mathrm{PCP}}^{d, \bullet}(X)} 1 d e=\int_{\operatorname{Sym}^{d}(B)}\left(\rho_{d}\right)_{*}(1) d e,
$$

where $\left(\rho_{d}\right)_{*}(1)$ is the $\mathbb{Z}((p))$-valued constructible function on $\operatorname{Sym}^{d}(B)$ given by pushing forward the Euler characteristic measure [12]. We denote $\left(\rho_{d}\right)_{*}(1)$ by $f_{d}$ so by definition, the value of $f_{d}$ at a point $\boldsymbol{a x}=\sum_{i} a_{i} x_{i} \in \operatorname{Sym}^{d}(B)$ is

$$
f_{d}(\boldsymbol{a x})=\int_{\rho_{d}^{-1}(\boldsymbol{a x})} 1 d e .
$$

We will show that $f_{d}$ has some nice multiplicative properties. Let $B^{\text {sing }} \subset B$ be the points over which the fibers of $S \rightarrow B$ are singular. Note that $\# B^{\text {sing }}=e(S)$. Let $B^{\mathrm{sm}}=B-B^{\text {sing }}$.

Proposition 9. Let $x_{1}, \ldots, x_{n} \in B^{\mathrm{sm}}$ and $y_{1}, \ldots, y_{m} \in B^{\mathrm{sing}}$ and let $a_{1}, \ldots$, $a_{n}, b_{1}, \ldots, b_{m}$ be positive integers summing to $d$. Let $\boldsymbol{a x}$ and $\boldsymbol{b} \boldsymbol{y}$ denote $\sum_{i} a_{i} x_{i}$ and $\sum_{j} b_{j} y_{j}$ respectively. Then there exist $F_{1} \in p^{1 / 2} \mathbb{Z}[[p]], F_{2} \in \mathbb{Z}[[p]]$, and $g, h: \mathbb{N} \rightarrow \mathbb{Z}((p))$ such that

$$
f_{d}(\boldsymbol{a} \boldsymbol{x}+\boldsymbol{b} \boldsymbol{y})=F_{1}^{e(B)} \cdot F_{2}^{e(S)} \cdot G(\boldsymbol{a x}) \cdot H(\boldsymbol{b} \boldsymbol{y}),
$$

where

$$
G(\boldsymbol{a x})=\prod_{i=1}^{n} g\left(a_{i}\right), \quad H(\boldsymbol{b} \boldsymbol{y})=\prod_{j=1}^{m} h\left(b_{j}\right)
$$

This proposition follows from Proposition 16 which will be stated and proved in the next section.

COROLlary 10.

$$
\widehat{\mathrm{DT}}(X)=F_{1}^{e(B)} \cdot F_{2}^{e(S)} \cdot\left(\sum_{a=0}^{\infty} g(a) q^{a}\right)^{e(B)-e(S)} \cdot\left(\sum_{b=0}^{\infty} h(b) q^{b}\right)^{e(S)},
$$

where we have set $g(0)=h(0)=1$. 
Proof. We apply Proposition 9 to the computation of $\widehat{\mathrm{DT}}(X)$ as follows

$$
\begin{aligned}
\widehat{\mathrm{DT}}(X) & =\int_{\mathrm{Hilb}_{\overrightarrow{\mathrm{PCP}}(X)}^{\bullet \bullet}} 1 d e \\
& =\int_{\mathrm{Sym}^{\bullet}(B)} f \bullet d e \\
& =F_{1}^{e(B)} \cdot F_{2}^{e(S)} \cdot \int_{\mathrm{Sym}^{\bullet}\left(B^{\mathrm{sm})}\right.} G d e \cdot \int_{\mathrm{Sym}^{\bullet}\left(B^{\text {sing }}\right)} H d e .
\end{aligned}
$$

Applying Lemma 32 to this last equation yields the corollary.

To prove Proposition 9 and explicitly compute $F_{1}, F_{2}, g$, and $h$, we need a good understanding of the strata $\rho_{d}^{-1}(\boldsymbol{a} \boldsymbol{x}+\boldsymbol{b} \boldsymbol{y}) \subset \operatorname{Hilb}_{\mathrm{PCP}}^{d, \bullet}(X)$.

For any

$$
\begin{aligned}
\boldsymbol{x}=\left(x_{1}, \ldots, x_{n}\right), & \boldsymbol{y}=\left(y_{1}, \ldots, y_{m}\right), \\
\lambda=\left(\lambda^{(1)}, \ldots, \lambda^{(n)}\right), \quad \boldsymbol{\mu} & =\left(\mu^{(1)}, \ldots, \mu^{(m)}\right),
\end{aligned}
$$

we define an associated Cohen-Macaulay curve

$$
C_{\boldsymbol{x}, \boldsymbol{y}, \lambda, \boldsymbol{\mu}}=B \bigcup_{i=1}^{n}\left(\lambda^{(i)} F_{x_{i}}\right) \bigcup_{j=1}^{m}\left(\mu^{(j)} F_{y_{j}}\right) .
$$

From Theorem 8 we obtain the following decomposition of the fibers of $\rho_{d}$ in $K_{0}\left(\operatorname{Var}_{\mathbb{C}}\right)((p))$ :

$$
\begin{aligned}
\rho_{d}^{-1}(\boldsymbol{a x}+\boldsymbol{b} \boldsymbol{y}) & =\sum_{\lambda \vdash \boldsymbol{a}} \sum_{\boldsymbol{\mu} \vdash \boldsymbol{b}} p^{\chi\left(\mathcal{O}_{\left.C_{x, y, \lambda, \mu}\right)} \operatorname{Hilb}^{\bullet}\left(X, C_{\boldsymbol{x}, \boldsymbol{y}, \lambda, \boldsymbol{\mu}}\right)\right.} \\
& =\sum_{\lambda \vdash \boldsymbol{a}} \sum_{\boldsymbol{\mu} \vdash \boldsymbol{b}} p^{\chi\left(\mathcal{O}_{\left.C_{x, y, \lambda, \mu}\right)} \operatorname{Quot}_{X}^{\bullet}\left(I_{C_{x, y, \lambda, \mu}}\right),\right.}
\end{aligned}
$$

where the second equality follows from Lemma 5. Here

$$
\boldsymbol{a}=\left(a_{1}, \ldots, a_{n}\right), \quad \boldsymbol{b}=\left(b_{1}, \ldots, b_{m}\right)
$$

and the meaning of $\boldsymbol{\lambda} \vdash \boldsymbol{a}$ and $\boldsymbol{\mu} \vdash \boldsymbol{b}$ is that $\lambda^{(i)} \vdash a_{i}$ and $\mu^{(j)} \vdash b_{j}$ for all $i$ and $j$. For later use, we state the following:

LEMMA 11. Let

$$
C_{x, y, \lambda, \mu}:=B \cup_{i}\left(\lambda^{(i)} F_{x_{i}}\right) \cup_{j}\left(\mu^{(j)} F_{y_{j}}\right)
$$

then

$$
\chi\left(\mathcal{O}_{C_{x, y, \lambda, \mu}}\right)=\chi\left(\mathcal{O}_{B}\right)-\sum_{i=1}^{n} \lambda_{1}^{(i)}-\sum_{j=1}^{m} \mu_{1}^{(j)}
$$


Proof. Since $\lambda^{(i)} F_{x_{i}}=p^{-1}\left(Z_{\lambda^{(i)}}\right)$ and $p$ is an elliptic fibration, $\chi\left(\mathcal{O}_{F_{x_{i}}}\right)=0$ and similarly we have $\chi\left(\mathcal{O}_{F_{y_{j}}}\right)=0$. Note that $B \cap \lambda^{(i)} F_{x_{i}}$ and $B \cap \mu^{(j)} F_{y_{j}}$ are zerodimensional subschemes of length $\lambda_{1}^{(i)}$ and $\mu_{1}^{(j)}$ respectively (see equation (1)). The lemma then follows from the exact sequence

$$
0 \rightarrow \mathcal{O}_{C} \rightarrow \mathcal{O}_{B} \oplus_{i} \mathcal{O}_{\lambda^{(i)} F_{x_{i}}} \oplus_{j} \mathcal{O}_{\mu^{(j)} F_{y_{j}}} \rightarrow \oplus_{i} \mathcal{O}_{B \cap \lambda^{(i)} F_{x_{i}}} \oplus_{j} \mathcal{O}_{B \cap \mu^{(j)} F_{y_{j}}} \rightarrow 0
$$

In the next section, we will see that the Euler characteristic of the Quot scheme $\operatorname{Quot}_{X}^{\bullet}\left(I_{C_{x, y, \lambda, \mu}}\right)$ does not depend on the exact location of the points $x_{i} \in B^{\mathrm{sm}}$ and $y_{j} \in B^{\text {sing }}$, but only on their number $n$ and $m$ and the partitions $\lambda^{(i)}$ and $\mu^{(j)}$.

\section{Stratifying according to embedded points}

In the previous two sections, we reduced our consideration to the strata $\operatorname{Quot}_{X}^{\bullet}\left(I_{C_{x, y, \lambda, \mu}}\right)$ of $\operatorname{Hilb}_{\mathrm{PCP}}^{d, \bullet}(X)$ which parameterize subschemes $Z$ whose maximal Cohen-Macaulay subscheme $Z_{\mathrm{CM}} \subset Z$ is the partition thickened comb curve

$$
C_{x, y, \lambda, \mu}:=B \cup_{i}\left(\lambda^{(i)} F_{x_{i}}\right) \cup_{j}\left(\mu^{(j)} F_{y_{j}}\right) .
$$

In this section, we introduce a further stratification of $\operatorname{Quot}_{X}^{\bullet}\left(I_{C_{x, y, \lambda, \mu}}\right)$ by keeping track of the support of the quotients with respect to the geometry of the underlying reduced curve $B \cup_{i} F_{x_{i}} \cup_{j} F_{y_{j}}$. This allows us to write $\operatorname{Quot}_{X}^{\bullet}\left(I_{C_{x, y, \lambda, \mu}}\right)$ as a product of 'local' Hilbert schemes (in $\left.K_{0}\left(\operatorname{Var}_{\mathbb{C}}\right)((p))\right)$. We then use this product to compute its Euler characteristic. The main result of this section is Proposition 16.

5.1. Stratification of $\boldsymbol{X}$. Given a Cohen-Macaulay curve $C_{\boldsymbol{x}, \boldsymbol{y}, \lambda, \boldsymbol{\mu}}$, its reduced support is given by $B \cup_{i} F_{x_{i}} \cup_{j} F_{y_{j}}$ which is a nodal curve with nodes at $\left(x_{1}, \ldots\right.$, $\left.x_{n}\right),\left(y_{1}, \ldots, y_{m}\right)$, and $\left(z_{1}, \ldots, z_{m}\right)$ where $z_{j}$ is the node of the nodal fiber $F_{y_{j}}$ (see Figure 1). Consider the following associated chain of closed subsets of $X$ :

$$
\cup_{i}\left\{x_{i}\right\} \cup_{j}\left\{y_{j}, z_{j}\right\} \subset B \cup_{i} F_{x_{i}} \cup_{j} F_{y_{j}} \subset X .
$$

This gives the following stratification of $X$ by locally closed subsets:

- $\cup_{i}\left\{x_{i}\right\} \cup_{j}\left\{y_{j}, z_{j}\right\}$;

- $B^{\circ}:=B \backslash \cup_{i}\left\{x_{i}\right\} \cup_{j}\left\{y_{j}\right\}$;

- $F_{x_{i}}^{\circ}:=F_{x_{i}} \backslash\left\{x_{i}\right\}$; 
- $F_{y_{j}}^{\circ}:=F_{y_{j}} \backslash\left\{y_{j}, z_{j}\right\}$

- $W:=X \backslash B \cup_{i} F_{x_{i}} \cup_{j} F_{y_{j}}$.

We denote the collection of these locally closed subsets by $\Sigma_{x, y, \lambda, \mu}$.

DEFINITION 12. Let $X$ be a smooth quasiprojective variety, $\mathcal{F}$ a coherent sheaf

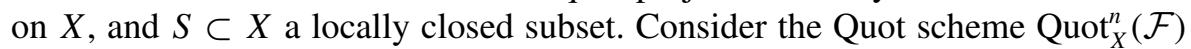
of quotients $\mathcal{F} \rightarrow Q$ on $X$, where $Q$ is zero-dimensional of length $n$. We define $\operatorname{Quot}_{X}^{n}(\mathcal{F}, S)$ as the locally closed subset of quotients $\mathcal{F} \rightarrow Q$ for which the reduced support of $Q$ lies in $S$.

We will use $\Sigma$ to provide a stratification of $\operatorname{Quot}_{X}^{\circ}\left(I_{C}\right)$ by locally closed subsets. For this, we need the following general result:

Proposition 13. Let $X$ be a smooth quasiprojective variety, $S \subset X$ a locally closed subset, $Z \subset X$ a closed subset, and $\mathcal{F}$ a coherent sheaf on $X$. Suppose $Z \subset S$. For any $n$, there exists a geometrically bijective constructible morphism

$$
\operatorname{Quot}_{X}^{n}(\mathcal{F}, S) \longrightarrow \bigsqcup_{n_{1}+n_{2}=n} \operatorname{Quot}_{X}^{n_{1}}(\mathcal{F}, S \backslash Z) \times \operatorname{Quot}_{X}^{n_{2}}(\mathcal{F}, Z)
$$

Proof. Denote by $X_{Z}^{(N)}$ the $(N-1)$ th order neighborhood of $Z \subset X$. That is, let $I_{Z} \subset \mathcal{O}_{X}$ be the ideal defining $Z \subset X$, then $X_{Z}^{(N)} \subset X$ is the closed subscheme defined by $I_{Z}^{N} \subset \mathcal{O}_{X}$. Denote the inclusion $X_{Z}^{(N)} \subset X$ by $\iota$ and let $U:=X \backslash Z$.

Fix $n$. We choose $N \gg 0$ with the following property. For any quotient $\mathcal{F} \rightarrow Q$, where $Q$ is zero-dimensional of length $\leqslant n$ and supported on $Z$, the schemetheoretic support of $Q$ is contained in $X_{Z}^{(N)}$.

We now describe the map of the proposition. Given a quotient $\mathcal{F} \rightarrow Q$ in $\operatorname{Quot}_{X}^{n}(\mathcal{F}, S)$, we obtain

$$
\begin{aligned}
\left.\mathcal{F}\right|_{U} & \left.\rightarrow Q\right|_{U} \\
\iota^{*} \mathcal{F} & \gg \iota^{*} Q .
\end{aligned}
$$

The trivial quotient $\left.\mathcal{F}\right|_{X \backslash(\text { Supp } Q \cap U)} \rightarrow 0$ and $\left.\left.\mathcal{F}\right|_{U} \rightarrow Q\right|_{U}$ glue on the overlap, so we obtain an element of $\operatorname{Quot}_{X}^{n_{1}}(\mathcal{F}, S \backslash Z)$ for some $n_{1} \leqslant n$. Moreover, push-forward along a closed embedding is exact, so we obtain an element of $\operatorname{Quot}_{X}^{n-n_{1}}(\mathcal{F}, Z)$ as follows

$$
\mathcal{F} \rightarrow \iota_{*} \iota^{*} \mathcal{F} \rightarrow \iota_{*} \iota^{*} Q
$$


We have to show that this constructible morphism is a bijection on $\mathbb{C}$-valued points. We start with injectivity. Suppose $\mathcal{F} \rightarrow Q_{i}$, for $i=1,2$, map to the same element. Then they agree on $U=X \backslash Z$. So it suffices to show that they agree on $X \backslash\left(\operatorname{Supp} Q_{1} \cap U\right)=X \backslash\left(\operatorname{Supp} Q_{2} \cap U\right)$. By hypothesis, we know that there exists an isomorphism

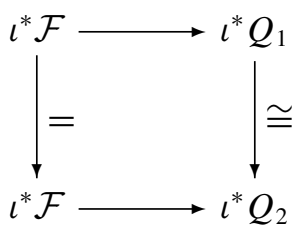

It suffices to show that pushing forward $\iota^{*} \mathcal{F} \rightarrow \iota^{*} Q_{i}$ to $X \backslash\left(\operatorname{Supp} Q_{1} \cap U\right)$ and composing with $\left.\left.\mathcal{F}\right|_{X \backslash\left(\operatorname{Supp} Q_{1} \cap U\right)} \rightarrow \iota_{*} \iota^{*} \mathcal{F}\right|_{X \backslash\left(\operatorname{Supp} Q_{1} \cap U\right)}$ gives back $\left.\left.\mathcal{F}\right|_{X \backslash\left(\operatorname{Supp} Q_{1} \cap U\right)} \rightarrow Q_{i}\right|_{X \backslash\left(\operatorname{Supp} Q_{1} \cap U\right)}$. This can be checked on an open affine cover.

Suppose $R$ is a commutative ring (corresponding to an open affine subset of $X \backslash\left(\operatorname{Supp} Q_{1} \cap U\right)$ ) and $I \subset R$ an ideal (corresponding to $Z$ ). Let $M \rightarrow$ $Q$ be a quotient of finitely generated $R$-modules with $Q$ zero-dimensional (corresponding to either of $\left.\left.\mathcal{F}\right|_{X \backslash\left(\text { Supp } Q_{1} \cap U\right)} \rightarrow Q_{i}\right|_{X \backslash\left(\text { Supp } Q_{1} \cap U\right)}$ ). By our choice of $N$, we have

$$
I^{N} \subset \operatorname{Ann}(Q) \subset I,
$$

where $\operatorname{Ann}(Q) \subset R$ denotes the annihilator ideal of $Q$. Consider the composition

$$
M \rightarrow M \otimes_{R} R / I^{N} \rightarrow Q \otimes_{R} R / I^{N},
$$

viewed as a morphism of $R$-modules, where the middle and third modules are $R$-modules via the map $R \rightarrow R / I^{N}$. Note that

$$
Q \otimes_{R} R / I^{N} \cong Q / I^{N} Q \cong Q
$$

since $I^{N} \subset \operatorname{Ann}(Q)$. Composing (4) with this isomorphism gives back the original quotient $M \rightarrow Q$.

For surjectivity, take two quotients $\mathcal{F} \rightarrow Q_{i}$, with $Q_{1}$ of length $n_{1}$ supported on $S \backslash Z$ and $Q_{2}$ of length $n-n_{1}$ supported on $Z$. Then $\left.\left.\mathcal{F}\right|_{U} \rightarrow Q_{1}\right|_{U}$ and $\left.\left.\mathcal{F}\right|_{X \backslash \text { Supp } Q_{1}} \rightarrow Q_{2}\right|_{X \backslash \text { Supp } Q_{1}}$ agree on the overlap. They glue to the required quotient $\mathcal{F} \rightarrow Q$, with $Q$ of length $n$ supported on $S$.

LEMMA 14. The following equation holds in $K_{0}\left(\operatorname{Var}_{\mathbb{C}}\right)((p))$

$$
\begin{aligned}
\operatorname{Quot}_{X}^{\bullet}\left(I_{C_{x, y, \lambda, \mu}}\right) & =\prod_{S \in \Sigma_{x, y, \lambda, \mu}} \operatorname{Quot}_{X}^{\bullet}\left(I_{C_{x, y, \lambda, \mu}}, S\right) \\
& =\operatorname{Quot}_{X}^{\bullet}\left(I_{C_{x, y, \lambda, \mu}}, W\right) \cdot \operatorname{Quot}_{X}^{\bullet}\left(I_{C_{x, y, \lambda, \mu}}, B^{\circ}\right)
\end{aligned}
$$




$$
\begin{aligned}
& \prod_{i=1}^{n} \operatorname{Quot}_{X}^{\bullet}\left(I_{C_{x, y, \lambda, \mu}},\left\{x_{i}\right\}\right) \cdot \operatorname{Quot}_{X}^{\bullet}\left(I_{C_{x, y, \lambda, \mu}}, F_{x_{i}}^{\circ}\right) \\
& \cdot \prod_{j=1}^{m} \operatorname{Quot}_{X}^{\bullet}\left(I_{C_{x, y, \lambda, \mu}},\left\{y_{j}\right\}\right) \cdot \operatorname{Quot}_{X}^{\bullet}\left(I_{C_{x, y, \lambda}, \mu},\left\{z_{j}\right\}\right)
\end{aligned}
$$

$$
\cdot \operatorname{Quot}_{X}^{\bullet}\left(I_{C_{x, y, \lambda, \mu}}, F_{y_{j}}^{\circ}\right) .
$$

Proof. Let $C:=C_{x, y, \lambda, \mu}$. First apply the previous proposition to $\operatorname{Quot}_{X}^{\bullet}\left(I_{C}\right)$ with $S=X$ and $Z=C_{\text {red }}$ in order to obtain

$$
\operatorname{Quot}_{X}^{\bullet}\left(I_{C}\right)=\operatorname{Quot}_{X}^{\bullet}\left(I_{C}, W\right) \cdot \operatorname{Quot}_{X}^{\bullet}\left(I_{C}, C_{\text {red }}\right) .
$$

Next, apply the previous proposition to $\operatorname{Quot}_{X}^{\bullet}\left(I_{C}, C_{\text {red }}\right)$ with $S=C_{\text {red }}$ and $Z=$ $F_{x_{1}}$ in order to obtain

$$
\operatorname{Quot}_{X}^{\bullet}\left(I_{C}\right)=\operatorname{Quot}_{X}^{\bullet}\left(I_{C}, W\right) \cdot \operatorname{Quot}_{X}^{\bullet}\left(I_{C}, C_{\text {red }} \backslash F_{x_{1}}\right) \cdot \operatorname{Quot}_{X}^{\bullet}\left(I_{C}, F_{x_{1}}\right) \text {. }
$$

Repeating this procedure, taking $S=C_{\text {red }} \backslash F_{x_{1}}$ and $Z=F_{x_{2}}$, and so on, we obtain

$$
\begin{aligned}
\operatorname{Quot}_{X}^{\bullet}\left(I_{C}\right)= & \operatorname{Quot}_{X}^{\bullet}\left(I_{C}, W\right) \cdot \operatorname{Quot}_{X}^{\bullet}\left(I_{C}, B^{\circ}\right) \\
& \cdot \prod_{i=1}^{n} \operatorname{Quot}_{X}^{\bullet}\left(I_{C}, F_{x_{i}}\right) \\
& \cdot \prod_{j=1}^{m} \operatorname{Quot}_{X}^{\bullet}\left(I_{C}, F_{y_{j}}\right) .
\end{aligned}
$$

Next, apply the previous proposition to $\operatorname{Quot}_{X}^{\bullet}\left(I_{C}, F_{x_{1}}\right), S=F_{x_{1}}$, and $Z=\left\{x_{1}\right\}$. Then

$$
\begin{aligned}
\operatorname{Quot}_{X}^{\bullet}\left(I_{C}\right)= & \operatorname{Quot}_{X}^{\bullet}\left(I_{C}, W\right) \cdot \operatorname{Quot}_{X}^{\bullet}\left(I_{C}, B^{\circ}\right) \\
& \cdot \operatorname{Quot}_{X}^{\bullet}\left(I_{C}, F_{x_{1}}^{\circ}\right) \cdot \operatorname{Quot}_{X}^{\bullet}\left(I_{C},\left\{x_{1}\right\}\right) \\
& \cdot \prod_{i=2}^{n} \operatorname{Quot}_{X}^{\bullet}\left(I_{C}, F_{x_{i}}\right) \\
& \cdot \prod_{j=1}^{m} \operatorname{Quot}_{X}^{\bullet}\left(I_{C}, F_{y_{j}}\right) .
\end{aligned}
$$

Repeating for all points $x_{2}, \ldots, x_{n}, y_{1}, \ldots, y_{m}, z_{1}, \ldots, z_{m}$, the required identity follows. 
5.2. Reduction to Quot schemes of $\mathbb{C}^{3}$. Let $\lambda, \mu, v$ be integer partitions which we also regard as subsets in $\left(\mathbb{Z}_{\geqslant 0}\right)^{2}$ by their diagram as in [6]. Consider the subscheme

$$
C_{\lambda \mu \nu}=C_{\lambda \varnothing \varnothing} \cup C_{\varnothing \mu \varnothing} \cup C_{\varnothing \varnothing \nu} \subset \mathbb{C}^{3}=\operatorname{Spec} \mathbb{C}[r, s, t]
$$

defined by the monomial ideal

$$
I_{\lambda \mu \nu}=I_{\lambda \varnothing \varnothing} \cap I_{\varnothing \mu \varnothing} \cap I_{\varnothing \varnothing \nu},
$$

where

$$
\begin{aligned}
r^{\rho} s^{\sigma} t^{\tau} \in I_{\lambda \varnothing \varnothing} & \Longleftrightarrow(\sigma, \tau) \notin \lambda, \\
r^{\rho} s^{\sigma} t^{\tau} \in I_{\varnothing \mu \varnothing} & \Longleftrightarrow(\tau, \rho) \notin \mu, \\
r^{\rho} s^{\sigma} t^{\tau} \in I_{\varnothing \varnothing \nu} & \Longleftrightarrow(\rho, \sigma) \notin v .
\end{aligned}
$$

Consider the Quot scheme Quot ${ }_{\mathbb{C}^{3}}^{n}\left(I_{C_{\lambda \mu \nu}}\right)$. Inside, we have the closed subset of quotients supported set-theoretically at the origin (Definition 12), which we denote by

$$
\operatorname{Quot}^{n}(\lambda, \mu, v):=\operatorname{Quot}_{\mathbb{C}^{3}}^{n}\left(I_{C_{\lambda \mu \nu}},\{0\}\right) .
$$

The kernel of such a quotient $I_{C_{\lambda \mu \nu}} \rightarrow Q$ is the ideal sheaf of a one-dimensional scheme $Z$ with underlying Cohen-Macaulay curve $C_{\lambda \mu \nu}$ and its embedded points supported set-theoretically at the origin. We emphasize that $Z$ need not be monomial.

We note that the permutations $(r, s, t) \mapsto(t, r, s)$ and $(r, s, t) \mapsto(s, r, t)$ induce the isomorphisms

$$
\operatorname{Quot}^{n}(\lambda, \mu, v) \cong \operatorname{Qut}^{n}(\nu, \lambda, \mu), \quad \operatorname{Quot}^{n}(\lambda, \mu, v) \cong \operatorname{Quot}^{n}\left(\mu^{\prime}, \lambda^{\prime}, v^{\prime}\right),
$$

where $\lambda^{\prime}=\{(\underset{\widetilde{V}}{i}, j):(j, i) \in \lambda\}$ denotes conjugate partition.

We define $\widetilde{\mathrm{V}}_{\lambda \mu \nu} \in \mathbb{Z}[[p]]$ by

$$
\widetilde{\mathrm{V}}_{\lambda \mu \nu}=e\left(\operatorname{Quot}^{\bullet}(\lambda, \mu, v)\right)
$$

and note the symmetries

$$
\widetilde{\mathbf{V}}_{\lambda \mu \nu}=\widetilde{\mathrm{V}}_{\nu \lambda \mu}=\widetilde{\mathrm{V}}_{\mu^{\prime} \lambda^{\prime} \nu^{\prime}}
$$

Recall that $S \subset X$ is the elliptic surface and $T=\operatorname{Tot}\left(\left.K_{S}\right|_{B}\right)$. For any point $p \in B$, let $R_{p}=\operatorname{Tot}\left(\left.K_{S}\right|_{F_{p}}\right)$. We choose local formal coordinates at $p=x_{i}$ or $p=y_{j}$ such that

$$
R_{p}=\{r=0\}, \quad S=\{s=0\}, \quad T=\{t=0\}
$$


and when $p$ is $z_{j}$

$$
R_{p}=\{r t=0\}, \quad S=\{s=0\} .
$$

Note that at $x_{i}$ or $y_{j}$, the curve $B$ is given by $\{s=t=0\}$ and the fiber $F_{x_{i}}$ or $F_{y_{j}}$ is given by $\{s=r=0\}$. At the point $z_{j}$, the fiber is a nodal curve and is given by $\{s=r t=0\}$.

Let $C_{\boldsymbol{x}, \boldsymbol{y}, \lambda, \boldsymbol{\mu}}$ be a Cohen-Macaulay curve as in the beginning of this section and consider the Quot schemes of Lemma 14.

LEMMA 15. We have the following equalities in $K_{0}\left(\operatorname{Var}_{\mathbb{C}}\right)$

$$
\begin{aligned}
& \operatorname{Quot}_{X}^{n}\left(I_{C_{x, y, \lambda, \mu}},\left\{x_{i}\right\}\right)=\operatorname{Quot}^{n}\left(\square, \varnothing, \lambda^{(i)}\right), \\
& \operatorname{Quot}_{X}^{n}\left(I_{C_{x, y, \lambda}, \mu},\left\{y_{j}\right\}\right)=\operatorname{Quot}^{n}\left(\square, \varnothing, \mu^{(j)}\right), \\
& \operatorname{Quot}_{X}^{n}\left(I_{C_{x, y, \lambda, \mu}},\left\{z_{j}\right\}\right)=\operatorname{Quot}^{n}\left(\mu^{(j) \prime}, \varnothing, \mu^{(j)}\right) .
\end{aligned}
$$

Here $\square$ is the unique partition of size 1 (whose diagram is a single box) and $\varnothing$ is the empty partition. Recall that the kernels of the quotients on the RHS do not need to be monomial ideals: although their underlying maximal Cohen-Macaulay subscheme is monomial, they may have arbitrary embedded points at the origin.

Proof. We prove the first equality; the others follow similarly. Let $C:=C_{\boldsymbol{x}, \boldsymbol{y}, \boldsymbol{\lambda}, \boldsymbol{\mu}}$. Take $N \gg 0$ such that for any quotient $I_{C} \rightarrow Q$, where $Q$ is set-theoretically supported at $\left\{x_{i}\right\}$ and of length $n$, the scheme-theoretic support of $Q$ lies inside the $(N-1)$ th order infinitesimal neighborhood $X_{\left\{x_{i}\right\}}^{(N)}$ of $\left\{x_{i}\right\}$. (Recall the definition of the closed subscheme $X_{Z}^{(N)}$ from the proof of Proposition 13.) Let $\iota: X_{\left\{x_{i}\right\}}^{(N)} \hookrightarrow X$ be the closed embedding. We have already seen in the proof of Proposition 13 that first restricting a quotient $I_{C} \rightarrow Q$ to $\iota^{*} I_{C} \rightarrow \iota^{*} Q$ and then pushing forward to $X$ loses no information. More precisely:

$$
I_{C} \rightarrow \iota_{*} \iota^{*} I_{C} \rightarrow \iota_{*} \iota^{*} Q
$$

is isomorphic to the original quotient $I_{C} \rightarrow Q$. Therefore, we have a geometric constructible morphism

$$
\operatorname{Quot}_{X}^{n}\left(I_{C},\left\{x_{i}\right\}\right) \rightarrow \operatorname{Quot}_{X_{\left\{x_{i}\right\}}^{(N)}}^{n}\left(\iota^{*} I_{C},\left\{x_{i}\right\}\right) .
$$

Similarly, we have a geometric constructible morphism

$$
\operatorname{Quot}_{\mathbb{C}^{3}}^{n}\left(I_{C_{\square \varnothing \lambda}(i)},\{0\}\right) \rightarrow \operatorname{Quot}_{\left(\mathbb{C}^{3}\right)_{\{0\}}^{(M)}}^{n}\left(I_{C_{\square \varnothing \lambda}(i)},\{0\}\right),
$$

where $\left(\mathbb{C}^{3}\right)_{\{0\}}^{(M)}$ is a large enough infinitesimal neighborhood of $0 \in \mathbb{C}^{3}$ and we may take $M=N \gg 0$. Since $X$ is smooth and three-dimensional, the formal 
completion of its local ring at $x_{i}$ is given by

$$
\widehat{\mathcal{O}}_{X, x_{i}} \cong \mathbb{C}[[r, s, t]] \text {. }
$$

Let $\mathfrak{m}_{x_{i}}$ be the maximal ideal corresponding to $x_{i}$. Then [1, Cor. 10.4]

$$
\widehat{\mathcal{O}}_{X, x_{i}} / \widehat{\mathfrak{m}}_{x_{i}}^{N} \cong \mathbb{C}[[r, s, t]] /(r, s, t)^{N} \cong \mathbb{C}[r, s, t] /(r, s, t)^{N} .
$$

Therefore $X_{\left\{x_{i}\right\}}^{(N)} \cong\left(\mathbb{C}^{3}\right)_{\{0\}}^{(N)}$ and, by our choice of coordinates $r, s$, $t$, we have $\iota^{*} I_{C} \cong$ $I_{C_{\square \varnothing \lambda(i)}}$. Hence

$$
\operatorname{Quot}_{X_{\left\{x_{i}\right\}}^{(N)}}^{n}\left(\iota^{*} I_{C},\left\{x_{i}\right\}\right) \cong \operatorname{Quot}_{\left(\mathbb{C}^{3}\right)_{\{0\}}^{(N)}}^{n}\left(I_{C_{\square \varnothing \lambda(i)}},\{0\}\right)
$$

and the required equality follows from the geometric bijections (6), (7).

A direct consequence of the above lemma and the symmetries of $\widetilde{V}$ is

$$
\begin{aligned}
& e\left(\operatorname{Quot}^{\bullet}\left(I_{C_{x, y, \lambda, \mu}},\left\{x_{i}\right\}\right)\right)=\widetilde{\mathrm{V}}_{\lambda^{(i)} \square \varnothing,}, \\
& e\left(\operatorname{Quot}^{\bullet}\left(I_{C_{x, y, \lambda, \mu}},\left\{y_{j}\right\}\right)\right)=\widetilde{\mathrm{V}}_{\mu^{(j)} \square \varnothing,}, \\
& e\left(\operatorname{Quot}^{\bullet}\left(I_{C_{x, y, \lambda, \mu}},\left\{z_{j}\right\}\right)\right)=\widetilde{\mathrm{V}}_{\mu^{(j)} \mu^{(j)} \varnothing}
\end{aligned}
$$

We also choose formal local coordinates at all other points. For each point in $B^{\circ}$, choose local coordinates $(r, s, t)$ such that $T=\{t=0\}$ and $S=\{s=0\}$. For each point in $F_{x_{i}}^{\circ}$ or $F_{y_{j}}^{\circ}$, choose local coordinates $(r, s, t)$ such that $S=\{s=0\}$ and $R_{x_{i}}=\{r=0\}$ or $R_{y_{j}}=\{r=0\}$ respectively. For each point in $W$, choose any formal local coordinates $(r, s, t)$.

Consider the following constructible support morphisms

$$
\begin{aligned}
& \sigma_{W}: \operatorname{Quot}_{X}^{\bullet}\left(I_{C_{x, y, \lambda, \mu}}, W\right) \rightarrow \operatorname{Sym}^{\bullet}(W), \\
& \sigma_{B^{\circ}}: \operatorname{Quot}_{X}^{\circ}\left(I_{C_{x, y, \lambda, \mu}}, B^{\circ}\right) \rightarrow \operatorname{Sym}^{\bullet}\left(B^{\circ}\right), \\
& \sigma_{F_{x_{i}}^{\circ}}: \operatorname{Quot}_{X}^{\circ}\left(I_{C_{x, y, \lambda, \mu}}, F_{x_{i}}^{\circ}\right) \rightarrow \operatorname{Sym}^{\bullet}\left(F_{x_{i}}^{\circ}\right), \\
& \sigma_{F_{y_{j}}^{\circ}}: \operatorname{Quot}_{X}^{\circ}\left(I_{C_{x, y, \lambda, \mu}}, F_{y_{j}}^{\circ}\right) \rightarrow \operatorname{Sym}^{\bullet}\left(F_{y_{j}}^{\circ}\right) .
\end{aligned}
$$

To each quotient $I_{C_{x, y, \lambda, \mu}} \rightarrow Q$ with kernel $I_{Z} \subset I_{C_{x, y, \lambda, \mu}}$, these maps assign the settheoretic support—weighted by length—of $I_{C_{x, y, \lambda, \mu}} / I_{Z}$, that is, the location and lengths of the embedded points or zero-dimensional components of $Z$.

Consider a point $p$ in $W, B^{\circ}, F_{x_{i}}^{\circ}$, or $F_{y_{j}}^{\circ}$. Then using the formal local coordinates chosen above, the same type of argument as in Lemma 15 gives the following equalities in $K_{0}\left(\operatorname{Var}_{\mathbb{C}}\right)$

$$
\sigma_{W}^{-1}(n p)=\operatorname{Quot}^{n}(\varnothing, \varnothing, \varnothing),
$$




$$
\begin{aligned}
& \sigma_{B^{\circ}}^{-1}(n p)=\operatorname{Quot}^{n}(\square, \varnothing, \varnothing), \\
& \sigma_{F_{x_{i}}^{\circ}}^{-1}(n p)=\operatorname{Quot}^{n}\left(\varnothing, \varnothing, \lambda^{(i)}\right), \\
& \sigma_{F_{y_{j}}^{\circ}}^{-1}(n p)=\operatorname{Quot}^{n}\left(\varnothing, \varnothing, \mu^{(j)}\right) .
\end{aligned}
$$

Using Proposition 13, we see that the pre-images of the support morphisms satisfy the following multiplicative property in $K_{0}\left(\operatorname{Var}_{\mathbb{C}}\right)((p))$

$$
\sigma_{U}^{-1}\left(\sum_{i} n_{i} p_{i}\right)=\prod_{i} \sigma_{U}^{-1}\left(n_{i} p_{i}\right) .
$$

Pushing forward the Euler characteristic measure along the support maps, applying Lemma 32, and using the symmetries of $\widetilde{V}$ we find the following formulas

$$
\begin{aligned}
e\left(\operatorname{Quot}_{X}^{\bullet}\left(I_{C_{x, y, \lambda, \mu}}, W\right)\right) & =\int_{\mathrm{Sym}^{\bullet}(W)}\left(\sigma_{W}\right)_{*}(1) d e=\left(\widetilde{\mathrm{V}}_{\varnothing \varnothing \varnothing}\right)^{e(W)} \\
e\left(\operatorname{Quot}_{X}^{\bullet}\left(I_{C_{x, y, \lambda, \mu}}, B^{\circ}\right)\right) & =\int_{\mathrm{Sym}^{\bullet}\left(B^{\circ}\right)}\left(\sigma_{\left.B^{\circ}\right)_{*}}(1) d e=\left(\widetilde{\mathrm{V}}_{\square \varnothing \varnothing}\right)^{e\left(B^{\circ}\right)},\right. \\
e\left(\operatorname{Quot}_{X}^{\bullet}\left(I_{C_{x, y, \lambda, \mu}}, F_{x_{i}}^{\circ}\right)\right) & =\int_{\mathrm{Sym}^{\bullet}\left(F_{x_{i}}^{\circ}\right)}\left(\sigma_{\left.F_{x_{i}}^{\circ}\right)_{*}}(1) d e=\left(\widetilde{\mathrm{V}}_{\lambda^{(i)} \varnothing \varnothing}\right)^{e\left(F_{x_{i}}^{\circ}\right)}\right. \\
e\left(\operatorname{Quot}_{X}^{\bullet}\left(I_{C_{x, y, \lambda, \mu}}, F_{y_{j}}^{\circ}\right)\right) & =\int_{\mathrm{Sym}^{\bullet}\left(F_{y_{j}}^{\circ}\right)}\left(\sigma_{F_{y_{j}}^{\circ}}\right)_{*}(1) d e=\left(\widetilde{\mathrm{V}}_{\mu^{(j)} \varnothing \varnothing}\right)^{e\left(F_{y_{j}}^{\circ}\right)}
\end{aligned}
$$

We are now ready to prove the main result of this section:

PROPOSITION 16. Recall that $f_{d}=\left(\rho_{d}\right)_{*}(1) \in \mathbb{Z}((p))$ is the push-forward of the Euler characteristic measure by the map $\rho_{d}$. As before, let $x_{1}, \ldots, x_{n} \in B^{\mathrm{sm}}, y_{1}$, $\ldots, y_{m} \in B^{\text {sing }}$ and let $a_{1}, \ldots, a_{n}, b_{1}, \ldots, b_{m}$ be positive integers summing to $d$. Then

$$
f_{d}(\boldsymbol{a} \boldsymbol{x}+\boldsymbol{b} \boldsymbol{y})=\left(p^{1 / 2} \frac{\widetilde{\mathrm{V}}_{\square \varnothing \varnothing}}{\widetilde{\mathbf{V}}_{\varnothing \varnothing \varnothing}}\right)^{e(B)} \cdot \widetilde{\mathbf{V}}_{\varnothing \varnothing \varnothing}^{e(S)} \cdot \prod_{i=1}^{n} g\left(a_{i}\right) \prod_{j=1}^{m} h\left(b_{j}\right)
$$

where

$$
\begin{aligned}
& g(a)=\sum_{\lambda \vdash a} p^{-\lambda_{1}} \frac{\widetilde{\mathrm{V}}_{\varnothing \varnothing \varnothing} \widetilde{\mathrm{V}}_{\lambda \square \varnothing}}{\widetilde{\mathrm{V}}_{\square \varnothing \varnothing} \widetilde{\mathrm{V}}_{\lambda \varnothing \varnothing}}, \\
& h(b)=\sum_{\mu \vdash b} p^{-\mu_{1}} \frac{\widetilde{\mathrm{V}}_{\mu \mu^{\prime} \varnothing} \widetilde{\mathrm{V}}_{\mu \square \varnothing}}{\widetilde{\mathrm{V}}_{\square \varnothing \varnothing} \widetilde{\mathrm{V}}_{\mu \varnothing \varnothing}} .
\end{aligned}
$$


Note that this proves Proposition 9 and provides the values of the unknowns $g, h$ (as above) and $F_{1}, F_{2}$

$$
F_{1}=p^{1 / 2} \frac{\widetilde{\mathrm{V}}_{\square \varnothing \varnothing}}{\widetilde{\mathrm{V}}_{\varnothing \varnothing \varnothing}}, \quad F_{2}=\widetilde{\mathrm{V}}_{\varnothing \varnothing \varnothing .}
$$

Proof. We apply, in order, equation (3), Lemma 14, equations (8) and (9), and Lemma 11 to compute

$$
\begin{aligned}
& f_{d}(\boldsymbol{a} \boldsymbol{x}+\boldsymbol{b} \boldsymbol{y})=e\left(\rho_{d}^{-1}(\boldsymbol{a} \boldsymbol{x}+\boldsymbol{b} \boldsymbol{y})\right) \\
& =\sum_{\lambda \vdash \boldsymbol{a}} \sum_{\boldsymbol{\mu} \vdash \boldsymbol{b}} p^{\chi\left(\mathcal{O}_{\left.C_{x, y, \lambda, \mu}\right)}\right.} e\left(\operatorname{Quot}_{X}^{\bullet}\left(I_{C_{x, y, \lambda, \mu}}\right)\right) \\
& =\sum_{\lambda \vdash \boldsymbol{a}} \sum_{\boldsymbol{\mu} \vdash \boldsymbol{b}} p^{\chi\left(\mathcal{O}_{\left.C_{x, y, \lambda, \mu}\right)}\right)} \cdot e\left(\operatorname{Quot}_{X}^{\bullet}\left(I_{C_{x, y, \lambda, \mu}}, W\right)\right) \cdot e\left(\operatorname{Quot}_{X}^{\bullet}\left(I_{C_{x, y, \lambda, \mu}}, B^{\circ}\right)\right. \\
& \cdot \prod_{i=1}^{n} e\left(\operatorname{Quot}_{X}^{\bullet}\left(I_{C_{x, y, \lambda, \mu}},\left\{x_{i}\right\}\right)\right) \cdot e\left(\operatorname{Quot}_{X}^{\bullet}\left(I_{C_{x, y, \lambda, \mu}}, F_{x_{i}}^{\circ}\right)\right. \\
& \cdot \prod_{j=1}^{m} e\left(\operatorname{Quot}_{X}^{\bullet}\left(I_{C_{x, y, \lambda, \mu}},\left\{y_{j}\right\}\right)\right) \cdot e\left(\operatorname{Quot}_{X}^{\bullet}\left(I_{C_{x, y, \lambda, \mu}},\left\{z_{j}\right\}\right)\right) \\
& \cdot e\left(\operatorname{Quot}_{X}^{\bullet}\left(I_{C_{x, y, \lambda, \mu}}, F_{y_{j}}^{\circ}\right)\right. \\
& =\widetilde{\mathrm{V}}_{\varnothing \varnothing \varnothing}^{e(W)} \cdot \widetilde{\mathrm{V}}_{\square \varnothing \varnothing}^{e\left(B^{\circ}\right)} \cdot \sum_{\lambda \vdash \boldsymbol{a}} \sum_{\boldsymbol{\mu} \vdash \boldsymbol{b}} p^{\chi\left(\mathcal{O}_{\left.C_{x, y, \lambda, \boldsymbol{\mu}}\right)}\right.} \\
& \cdot \prod_{i=1}^{n} \widetilde{\mathrm{V}}_{\lambda^{(i)} \square \varnothing} \cdot \widetilde{\mathrm{V}}_{\lambda^{(i)}\left(F_{i}^{\circ}\right)}^{\circ} \cdot \prod_{j=1}^{m} \widetilde{\mathrm{V}}_{\mu^{(j)} \square \varnothing} \cdot \widetilde{\mathrm{V}}_{\mu^{(j)} \mu^{(j)^{\prime}} \varnothing} \cdot \widetilde{\mathrm{V}}_{\mu^{(j)} \varnothing \varnothing}^{e\left(F_{y_{j}}^{\circ}\right)} \\
& =p^{\chi\left(\mathcal{O}_{B}\right)} \cdot \widetilde{\mathbf{V}}_{\varnothing \varnothing \varnothing}^{e(W)} \cdot \widetilde{\mathbf{V}}_{\square \varnothing \varnothing}^{e\left(B^{\circ}\right)} \\
& \cdot \prod_{i=1}^{n} \sum_{\lambda^{(i)} \vdash a_{i}} p^{-\lambda_{1}^{(i)}} \widetilde{\mathrm{V}}_{\lambda^{(i)} \square \varnothing} \cdot \widetilde{\mathrm{V}}_{\lambda^{(i)} \varnothing \varnothing}^{e\left(F_{x_{i}}^{\circ}\right)} \\
& \cdot \prod_{j=1}^{m} \sum_{\mu^{(j)} \vdash b_{j}} p^{-\mu_{1}^{(j)}} \widetilde{\mathrm{V}}_{\mu^{(j)} \square \varnothing} \cdot \widetilde{\mathrm{V}}_{\mu^{(j)} \mu^{(j)} \varnothing} \cdot \widetilde{\mathrm{V}}_{\mu^{(j)} \varnothing \varnothing}^{e\left(F_{y_{j}}^{\circ}\right)} .
\end{aligned}
$$

We note that $e\left(F_{x_{i}}\right)=0$ and $e\left(F_{y_{j}}\right)=1$ so that $e\left(F_{x_{i}}^{\circ}\right)=-1$ and $e\left(F_{y_{j}}^{\circ}\right)=-1$. Also, since $e\left(B^{\circ}\right)=e(B)-n-m$, we have

$$
\begin{aligned}
e(W) & =e(S)-e\left(B^{\circ}\right)-\sum_{i} e\left(F_{x_{i}}\right)-\sum_{j} e\left(F_{y_{j}}\right) \\
& =e(S)-e(B)+n .
\end{aligned}
$$


The above equations allow us to redistribute the terms of $f_{d}(\boldsymbol{a} \boldsymbol{x}+\boldsymbol{b} \boldsymbol{y})$ as follows

$$
\begin{aligned}
& f_{d}(\boldsymbol{a} \boldsymbol{x}+\boldsymbol{b} \boldsymbol{y})=p^{\chi\left(\mathcal{O}_{B}\right)} \cdot \widetilde{\mathrm{V}}_{\varnothing \varnothing \varnothing(S)}^{e(} \cdot\left(\frac{\widetilde{\mathrm{V}}_{\square \varnothing \varnothing}}{\widetilde{\mathrm{V}}_{\varnothing \varnothing \varnothing}}\right)^{e(B)} \\
& \cdot \prod_{i=1}^{n} \sum_{\lambda^{(i)} \vdash a_{i}} p^{-\lambda_{1}^{(i)}} \cdot \frac{\widetilde{\mathrm{V}}_{\varnothing \varnothing \varnothing} \widetilde{\mathrm{V}}_{\lambda^{(i)} \square \varnothing}}{\widetilde{\mathrm{V}}_{\square \varnothing \varnothing} \widetilde{\mathrm{V}}_{\lambda^{(i)} \varnothing \varnothing}} \\
& \cdot \prod_{j=1}^{m} \sum_{\mu^{(j)} \vdash b_{j}} p^{-\mu_{1}^{(j)}} \cdot \frac{\widetilde{\mathbf{V}}_{\mu^{(j)} \mu^{(j)} \varnothing} \widetilde{\mathbf{V}}_{\mu^{(j)} \square \varnothing}}{\widetilde{\mathrm{V}}_{\square \varnothing \varnothing} \widetilde{\mathbf{V}}_{\mu^{(j)} \varnothing \varnothing}} .
\end{aligned}
$$

Noting that $\chi\left(\mathcal{O}_{B}\right)=e(B) / 2$, we see the above proves the proposition.

\section{Reduction to the topological vertex}

In this section, we express $\widehat{\mathrm{DT}}(X)$ in terms of the topological vertex, and then use the trace formulas of [6] to obtain a closed formula for $\widehat{\mathrm{DT}}(X)$.

6.1. $\tilde{\mathbf{V}}_{\lambda \mu \nu}$ in terms of $\mathbf{V}_{\lambda \mu \nu}$. Recall that the coefficients of the series $\widetilde{\mathbf{V}}_{\lambda \mu \nu} \in$ $\mathbb{Z}[[p]]$ are given by the Euler characteristics of Quot schemes on $\mathbb{C}^{3}$

$$
\widetilde{\mathrm{V}}_{\lambda \mu \nu}=e\left(\operatorname{Quot}_{\mathbb{C}^{3}}^{\bullet}\left(I_{C_{\lambda \mu \nu}}\right)\right) \text {. }
$$

We can compute the Euler characteristics using the $T=\left(\mathbb{C}^{*}\right)^{3}$-action on the Quot schemes induced by the $T$-action on $\mathbb{C}^{3}$. An ideal $I \subset \mathbb{C}[r, s, t]$ is $T$-invariant if and only if it is generated by monomials (such as $I_{C_{\lambda \mu \nu}}$ ). Moreover, there is a bijection between monomial ideals and 3D-partitions (see [6, § 6.3]) where a monomial ideal $I \subset \mathbb{C}[r, s, t]$ corresponds to a 3D-partition $\pi \in\left(\mathbb{Z}_{\geqslant 0}\right)^{3}$ by

$$
(\rho, \sigma, \tau) \in \pi \quad \Longleftrightarrow r^{\rho} s^{\sigma} t^{\tau} \notin I \text {. }
$$

The subschemes represented by points in $\operatorname{Quot}_{\mathbb{C}^{3}}^{\bullet}\left(I_{C_{\lambda \mu \nu}}\right)^{T}$ are given by quotients $I_{C_{\lambda \mu \nu}} \rightarrow Q$ with kernel equal to a monomial ideal corresponding to a 3D-partition asymptotic to $(\lambda \mu \nu)$, see [6, Defn 1]. Consequently,

$$
\begin{aligned}
\widetilde{\mathrm{V}}_{\lambda \mu \nu} & =e\left(\operatorname{Quot}_{\mathbb{C}^{3}}^{\bullet}\left(I_{C_{\lambda \mu \nu}}\right)^{T}\right) \\
& =\sum_{\pi} p^{n(\pi)},
\end{aligned}
$$

where the sum runs over all 3D-partitions asymptotic to $(\lambda \mu \nu)$ and $n(\pi)$ is the number of boxes in $\pi$ which are not contained in any of the legs. We see that $\widetilde{V}_{\lambda \mu \nu}$ 
differs from the usual topological vertex by an overall normalization

$$
\mathrm{V}_{\lambda \mu \nu}=p^{\left|\pi_{\min }\right|} \widetilde{\mathrm{V}}_{\lambda \mu \nu}
$$

where $\mathrm{V}_{\lambda \mu \nu}$ is the usual topological vertex [6, Defn 2] and $\left|\pi_{\min }\right|$ is the renormalized volume [6, page 2] of the minimal 3D-partition asymptotic to $(\lambda \mu \nu)$.

LEMMA 17. The following hold

$$
\mathrm{V}_{\lambda \varnothing \varnothing}=\widetilde{\mathrm{V}}_{\lambda \varnothing \varnothing}, \quad \mathrm{V}_{\lambda \square \varnothing}=p^{-\lambda_{1}} \widetilde{\mathrm{V}}_{\lambda \square \varnothing}, \quad \mathrm{V}_{\mu \mu^{\prime} \varnothing}=p^{-\|\mu\|^{2}} \widetilde{\mathrm{V}}_{\mu \mu^{\prime} \varnothing}
$$

where $\|\mu\|^{2}:=\sum_{j=1}^{\infty} \mu_{j}^{2}$.

Proof. The renormalized volume of a 3D-partition asymptotic to $(\lambda \mu \nu)$ is defined by

$$
|\pi|=\sum_{(\rho, \sigma, \tau) \in \pi}(1-\# \text { of legs of } \pi \text { containing }(\rho, \sigma, \tau)) .
$$

For $\pi_{\min }$, the minimal 3D-partition asymptotic to $(\lambda \square \varnothing)$, the only cubes contributing to $\left|\pi_{\min }\right|$ are those contained in both the $\square$-leg and the $\lambda$-leg. They intersect exactly in the cubes corresponding to the first part of $\lambda$, namely $\lambda_{1}$. Thus $\left|\pi_{\min }\right|=-\lambda_{1}$ in this case.

For the case of $(\lambda \varnothing \varnothing)$ every cube is in the $\lambda$-leg and so $\left|\pi_{\min }\right|=0$. For the case of $\left(\mu \mu^{\prime} \varnothing\right)$, each cube in the intersection of the $\mu$-leg and the $\mu^{\prime}$-leg contribute -1 and all other cubes contribute 0 . This intersection is a stack of squares of side lengths $\mu_{1}, \mu_{2}, \ldots$ and hence

$$
\left|\pi_{\min }\right|=-\sum_{j=1}^{\infty} \mu_{j}^{2}
$$

6.2. Applying the trace formulas. Substituting the values of $F_{1}, F_{2}, g$, and $h$ from Proposition 16 into Corollary 10, and then substituting in the formulas from Lemma 17 we obtain the following

$$
\begin{aligned}
\widehat{\mathrm{DT}}(X)= & \left(p^{1 / 2} \frac{\mathrm{V}_{\square \varnothing \varnothing}}{\mathrm{V}_{\varnothing \varnothing \varnothing}}\right)^{e(B)} \cdot \mathrm{V}_{\varnothing \varnothing \varnothing}^{e(S)} \cdot\left(\sum_{\lambda} \frac{\mathrm{V}_{\varnothing \varnothing \varnothing} \mathrm{V}_{\lambda \square \varnothing}}{\mathrm{V}_{\square \varnothing \varnothing} \mathrm{V}_{\lambda \varnothing \varnothing}} q^{|\lambda|}\right)^{e(B)-e(S)} \\
& \cdot\left(\sum_{\mu} p^{\|\mu\|^{2}} \frac{\mathrm{V}_{\mu \mu^{\prime} \varnothing} \mathrm{V}_{\mu \square \varnothing}}{\mathrm{V}_{\square \varnothing \varnothing} \mathrm{V}_{\mu \varnothing \varnothing}} q^{|\mu|}\right)^{e(S)} .
\end{aligned}
$$


From the Okounkov-Reshetikhin-Vafa formula for the topological vertex [16], [6, equation (5)] we get

$$
\mathrm{V}_{\varnothing \varnothing \varnothing}=M(p), \quad \mathrm{V}_{\square \varnothing \varnothing}=\frac{M(p)}{1-p},
$$

which we substitute into the above to find

$$
\begin{aligned}
\widehat{\mathrm{DT}}(X)= & \left(\frac{1}{p^{-1 / 2}-p^{1 / 2}}\right)^{e(B)}\left(\sum_{\lambda}(1-p) \frac{\mathrm{V}_{\lambda \square \varnothing}}{\mathrm{V}_{\lambda \varnothing \varnothing}} q^{|\lambda|}\right)^{e(B)-e(S)} \\
& \cdot\left(\sum_{\mu}(1-p) p^{\|\mu\|^{2}} \mathrm{~V}_{\mu \mu^{\prime} \varnothing} \frac{\mathrm{V}_{\mu \square \varnothing}}{\mathrm{V}_{\mu \varnothing \varnothing}} q^{|\mu|}\right)^{e(S)}
\end{aligned}
$$

Applying [6, equations (2)\&(4)], we see that

$$
\begin{aligned}
\widehat{\mathrm{DT}}(X)= & \left(p^{-1 / 2}-p^{1 / 2}\right)^{-e(B)} \cdot\left(\prod_{d=1}^{\infty} \frac{\left(1-q^{d}\right)}{\left(1-p q^{d}\right)\left(1-p^{-1} q^{d}\right)}\right)^{e(B)-e(S)} \\
& \cdot\left(M(p) \prod_{d=1}^{\infty} \frac{M\left(p, q^{d}\right)}{\left(1-p q^{d}\right)\left(1-p^{-1} q^{d}\right)}\right)^{e(S)} \cdot
\end{aligned}
$$

Noting that $e(B)$ is even, the above expression is easily seen to be equivalent to the formula for $\widehat{\mathrm{DT}}(X)$ in Theorem 1. Since the formula for $\widehat{\mathrm{DT}}_{\mathrm{fib}}(X)$ was previously proven by Toda, we may now regard the proof of Theorem 1 complete. In the next section, we will outline the proof of the formula for $\widehat{\mathrm{DT}}_{\text {fib }}(X)$ using our methods.

\section{The case of $\widehat{\mathrm{DT}}_{\text {fib }}(X)$}

The formula for $\widehat{\mathrm{DT}}_{\text {fib }}(X)$ given in Theorem 1 follows from a wall-crossing computation of Toda [18, Theorem 6.9] along with the PT/DT correspondence [4]. However, in this section we describe how to adapt our computation of $\widehat{\mathrm{DT}}(X)$ to the easier case of $\widehat{D T}_{\text {fib }}(X)$. Our approach yields a proof which is independent of Toda's.

DEFINITION 18. We say that a subscheme $C \subset X$ is a partition thickened fiber curve if it is of the form

$$
C=\cup_{i}\left(\lambda^{(i)} F_{x_{i}}\right)
$$

where we are using the notation of Definition 7 . We say that a subscheme $Z \subset X$ is a partition thickened fiber curve with points (PFP) if the maximal 
Cohen-Macaulay subscheme $Z_{\mathrm{CM}} \subset Z$ is a partition thickened fiber curve. We denote by

$$
\operatorname{Hilb}_{\mathrm{PFP}}^{d F, n}(X) \subset \operatorname{Hilb}^{d F, n}(X)
$$

the locus in the Hilbert scheme parameterizing PFP.

Our proof of Theorem 8 is easily adapted to prove the following:

THEOREM 19. If a subscheme $Z \subset X$ in the class $[Z]=d F$ is $\mathbb{C}^{*}$-invariant, then it is a PFP. That is

$$
\operatorname{Hilb}^{d F, n}(X)^{\mathbb{C}^{*}} \subset \operatorname{Hilb}_{\mathrm{PFP}}^{d F, n}(X) \subset \operatorname{Hilb}^{d F, n}(X) .
$$

Moreover, $\mathbb{C}^{*}$ acts on $\operatorname{Hilb}_{\mathrm{PFP}}^{d F, n}(X)$ and there exists a constructible morphism

$$
\rho_{d}^{\mathrm{fib}}: \operatorname{Hilb}_{\mathrm{PFP}}^{d F, \bullet}(X) \rightarrow \operatorname{Sym}^{d}(B)
$$

such that if $[Z] \in \operatorname{Hilb}_{\mathrm{PFP}}^{d F, n}(X)$, where the maximal Cohen-Macaulay subscheme of $Z$ is $\cup_{i}\left(\lambda^{(i)} F_{x_{i}}\right)$, then

$$
\rho_{d}^{\mathrm{fib}}([Z])=\sum_{i}\left|\lambda^{(i)}\right| x_{i}
$$

Define the following Cohen-Macaulay curve

$$
C_{\boldsymbol{x}, \boldsymbol{y}, \lambda, \boldsymbol{\mu}}^{\mathrm{fib}}:=\bigcup_{i=1}^{n}\left(\lambda^{(i)} F_{x_{i}}\right) \bigcup_{j=1}^{m}\left(\mu^{(j)} F_{y_{j}}\right) .
$$

Similar to equation (3), the previous theorem implies that the pre-images of points under the map $\rho_{d}^{\text {fib }}$ break into components

$$
\left(\rho_{d}^{\mathrm{fib}}\right)^{-1}(\boldsymbol{a x}+\boldsymbol{b} \boldsymbol{y})=\sum_{\lambda \vdash \boldsymbol{a}} \sum_{\mu \vdash \boldsymbol{b}} p^{\chi\left(\mathcal{O}_{C_{\boldsymbol{x}, y, \lambda, \mu}^{\mathrm{fib}}}\right)} \operatorname{Quot}_{X}^{\bullet}\left(I_{C_{\boldsymbol{x}, \boldsymbol{y}, \lambda, \mu}^{\mathrm{fib}}}\right),
$$

where we have adopted the same notation as in Section 4. Since the only nodes of the reduced support of $C_{\boldsymbol{x}, \boldsymbol{y}, \lambda, \boldsymbol{\mu}}^{\mathrm{fib}}$ are $z_{1}, \ldots, z_{m}$ (adopting the notation of Section 5) our stratification is simpler in this case

$$
\Sigma_{\boldsymbol{x}, \boldsymbol{y}, \lambda, \boldsymbol{\mu}}^{\mathrm{fib}}=\left\{\left\{z_{1}\right\}, \ldots,\left\{z_{m}\right\}, F_{y_{1}}^{\circ}, \ldots, F_{y_{m}}^{\circ}, F_{x_{1}}, \ldots, F_{x_{n}}, W\right\},
$$

where this time

$$
F_{y_{j}}^{\circ}=F_{y_{j}}-\left\{z_{j}\right\}, \quad W=X-\left(\cup_{i} F_{x_{i}} \cup_{j} F_{y_{j}}\right) .
$$

With virtually the same proof, we obtain the following analog of Lemma 14: 
LEMMA 20. The following equation holds in $K_{0}\left(\operatorname{Var}_{\mathbb{C}}\right)((p))$

$$
\begin{aligned}
\operatorname{Quot}_{X}^{\bullet}\left(I_{C_{x, y, \lambda, \mu}^{\mathrm{fib}}}\right)= & \operatorname{Quot}_{X}^{\bullet}\left(I_{C_{x, y, \lambda, \mu}^{\mathrm{fib}}}, W\right) \cdot \prod_{i=1}^{n} \operatorname{Quot}_{X}^{\bullet}\left(I_{C_{x, y, \lambda, \mu}^{\mathrm{fib}},}, F_{x_{i}}\right) \\
& \cdot \prod_{j=1}^{m} \operatorname{Quot}_{X}^{\bullet}\left(I_{C_{x, y, \lambda, \mu}^{\mathrm{fib}},}, F_{y_{j}}^{\circ}\right) \cdot \operatorname{Quot}_{X}^{\bullet}\left(I_{C_{x, y, \lambda, \mu}^{\mathrm{fib}}},\left\{z_{j}\right\}\right) .
\end{aligned}
$$

We choose the same set of formal local coordinates at each point as we did in Section 5 and by the same reasoning as in that section, we find

$$
\begin{aligned}
e\left(\operatorname{Quot}_{X}^{\bullet}\left(I_{C_{x, y, \lambda, \mu}^{\mathrm{fib}},},\left\{z_{j}\right\}\right)\right) & =\widetilde{\mathrm{V}}_{\mu^{(j)} \mu^{(j) \prime} \varnothing}, \\
e\left(\operatorname{Quot}_{X}^{\bullet}\left(I_{C_{x, y, \lambda, \mu}^{\mathrm{fib}}}, W\right)\right) & =\widetilde{\mathrm{V}}_{\varnothing \varnothing \varnothing \varnothing}^{e(W)}, \\
e\left(\operatorname{Quot}_{X}^{\bullet}\left(I_{C_{x, y, \lambda, \mu}^{\mathrm{fib}},}, F_{x_{i}}\right)\right) & =\widetilde{\mathrm{V}}_{\lambda^{(i)} \varnothing \varnothing}^{e\left(F_{x_{i}}\right)}, \\
e\left(\operatorname{Quot}_{X}^{\bullet}\left(I_{C_{x, y, \lambda, \mu}^{\mathrm{fib}},}, F_{y_{j}}^{\circ}\right)\right) & =\widetilde{\mathrm{V}}_{\mu^{(j)} \varnothing \varnothing}^{e\left(F_{y_{j}}^{\circ}\right)} .
\end{aligned}
$$

Now since $\chi\left(\mathcal{O}_{C_{x, y, \lambda, \mu}^{\mathrm{fib}}}\right)=0, e\left(F_{x_{i}}\right)=e\left(F_{y_{j}}^{\circ}\right)=0$, and

$$
e(W)=e(S)-m
$$

we have that

$$
\begin{aligned}
f_{d}^{\mathrm{fib}}(\boldsymbol{a x}+\boldsymbol{b} \boldsymbol{y}) & :=e\left(\left(\rho_{d}^{\mathrm{fib}}\right)^{-1}(\boldsymbol{a x}+\boldsymbol{b} \boldsymbol{y})\right) \\
& =\sum_{\lambda \vdash \boldsymbol{a}} \sum_{\boldsymbol{\mu} \vdash \boldsymbol{b}} p^{\chi\left(\mathcal{O}_{C_{\boldsymbol{x}, \boldsymbol{y}, \lambda, \mu}}\right)} e\left(\operatorname{Quot}_{X}^{\bullet}\left(I_{C_{\boldsymbol{x}, \boldsymbol{y}, \lambda, \boldsymbol{\mu}}^{\mathrm{fib}}}\right)\right) \\
& =\sum_{\lambda \vdash \boldsymbol{a}} \sum_{\boldsymbol{\mu} \vdash \boldsymbol{b}} \widetilde{\mathrm{V}}_{\varnothing \varnothing \varnothing}^{e(S)} \prod_{j=1}^{m} \frac{\widetilde{\mathrm{V}}_{\mu^{(j)} \mu^{(j)^{\prime} \varnothing}}}{\widetilde{\mathrm{V}}_{\varnothing \varnothing \varnothing}} .
\end{aligned}
$$

We may rewrite the above as

$$
f_{d}^{\mathrm{fib}}(\boldsymbol{a} \boldsymbol{x}+\boldsymbol{b} \boldsymbol{y})=\widetilde{\mathbf{V}}_{\varnothing \varnothing \varnothing}^{e(S)} \prod_{i=1}^{n} g_{\mathrm{fib}}\left(a_{i}\right) \prod_{j=1}^{m} h_{\mathrm{fib}}\left(b_{j}\right),
$$

where

$$
g_{\text {fib }}(a)=\sum_{\lambda \vdash a} 1 \quad h_{\text {fib }}(b)=\sum_{\mu \vdash b} \frac{\widetilde{\mathrm{V}}_{\mu \mu^{\prime} \varnothing}}{\widetilde{\mathrm{V}}_{\varnothing \varnothing \varnothing}} .
$$

We then get the following result, analogous to Corollary 10, with a similar proof

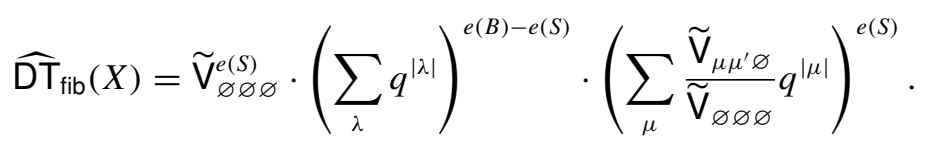


Substituting in the equations in Lemma 17, using the well-known generating function for 2D-partitions, and applying [6, equation (1)] we get

$$
\widehat{\mathrm{DT}}_{\mathrm{fib}}(X)=M(p)^{e(S)} \cdot\left(\prod_{d=1}^{\infty}\left(1-q^{d}\right)^{-1}\right)^{e(B)-e(S)} \cdot\left(\prod_{d=1}^{\infty}\left(1-q^{d}\right)^{-1} M\left(p, q^{d}\right)\right)^{e(S)}
$$

which is easily seen to be equivalent to the formula for $\widehat{\mathrm{DT}}_{\text {fib }}(X)$ given in Theorem 1.

\section{Including the Behrend function}

The aim of this section is to prove Theorem 3, which says that up to an overall sign, the partition functions $\widehat{\mathrm{DT}}(X)$ and $\mathrm{DT}(X)$ are equal after the simple change of variables $y=-p$. In order to do this we will need to assume a conjecture about the Behrend function which we formulate below for general Calabi-Yau threefolds and may be of independent interest.

Let $Y$ be any quasiprojective Calabi-Yau threefold. Let $C \subset Y$ be a (not necessarily reduced) Cohen-Macaulay curve with proper support. Assume that the singularities of $C_{\text {red }}$ are locally toric. (This means that formally locally $C_{\text {red }}$ is either smooth, nodal, or the union of the three coordinate axes. That is at $p \in C_{\text {red }} \subset Y$ the ideal $\widehat{I}_{C_{\text {red }}} \subset \widehat{\mathcal{O}}_{Y, p}$ is given by $\left(x_{1}, x_{2}\right),\left(x_{1}, x_{2} x_{3}\right)$, or $\left(x_{1} x_{2}, x_{2} x_{3}\right.$, $\left.x_{1} x_{3}\right)$ for some isomorphism $\widehat{\mathcal{O}}_{Y, p} \cong \mathbb{C}\left[\left[x_{1}, x_{2}, x_{3}\right]\right]$.) Recall that by Definition 4

$\operatorname{Hilb}^{n}(Y, C)=\left\{Z \subset Y\right.$ such that $C \subset Z$ and $I_{C} / I_{Z}$ has finite length $\left.n\right\}$.

Note that $\operatorname{Hilb}^{n}(Y, C) \subset \operatorname{Hilb}(Y)$ and let $v$ denote the Behrend function on $\operatorname{Hilb}(Y)$. Our conjecture is the following:

CONJECTURE 21.

$$
\int_{\operatorname{Hilb}^{n}(Y, C)} v d e=(-1)^{n} v([C]) \int_{\operatorname{Hilb}^{n}(Y, C)} d e,
$$

where $v([C])$ is the value of the Behrend function at the point $[C] \in \operatorname{Hilb}(Y)$.

REMARK 22. Conceivably, the condition that $C_{\text {red }}$ has locally toric singularities could be weakened, although we do not have any evidence for this case. Our conjecture is true for $Y$ a (globally) toric Calabi-Yau, with torus $T$, and $C \subset Y$ any $T$-fixed Cohen-Macaulay curve. In this case both sides of our conjecture can be computed by restricting to the $T$-fixed points, which are isolated. The Behrend function at such a fixed point $P$ is given by $v(P)=(-1)^{\operatorname{dim} T_{P} \operatorname{Hilb}(Y)}$, 
[3, Theorem 3.4]. The calculation of $(-1)^{\operatorname{dim} T_{P} \operatorname{Hilb}(Y)}$ is done in [13]; specifically, the left hand side of the equation in [13, Theorem 2] is easily seen to be $(-1)^{\operatorname{dim} T_{P} \operatorname{Hilb}(Y)}$ while the RHS clearly obeys the formula in our conjecture.

One could also make the much stronger conjecture that

$$
v([Z])=(-1)^{n} v([C]),
$$

for all $[Z] \in \operatorname{Hilb}^{n}(Y, C)$. This would of course imply our conjecture as stated. However, we do not know whether this stronger version holds, even in the case where $Y$ is $\mathbb{C}^{3}$ and $C$ is empty. In this case, this stronger conjecture says that the Behrend function on $\operatorname{Hilb}^{n}\left(\mathbb{C}^{3}\right)$ is the constant function $(-1)^{n}$.

8.1. Proof of Theorem 3. The Behrend function of any scheme is invariant under automorphisms. In particular, it is constant on the orbits of the $\mathbb{C}^{*}$ action on $\operatorname{Hilb}(X)$. We thus have

$$
\mathrm{DT}(X)=\int_{\mathrm{Hilb}^{\bullet \bullet \bullet}(X)} v d e=\int_{\mathrm{Hilb}^{\bullet \bullet \bullet}(X)^{\mathbb{C}^{*}}} v d e=\int_{\mathrm{Hilb}_{\mathrm{PCP}}(X)} v d e
$$

and so

$$
\operatorname{DT}(X)=\int_{\operatorname{Sym}^{\bullet}(B)}\left(\rho_{\bullet}\right)_{*}(v) d e .
$$

Let $f_{d}^{v}=\left(\rho_{d}\right)_{*}(\nu)$ so that in the notation of Section 4, we have

$$
f_{d}^{v}(\boldsymbol{a} \boldsymbol{x}+\boldsymbol{b} \boldsymbol{y})=\int_{\rho_{d}^{-1}(\boldsymbol{a} \boldsymbol{x}+\boldsymbol{b} \boldsymbol{y})} v d e .
$$

Recall that for the partition function $\mathrm{DT}(X)$, the variable tracking the holomorphic Euler characteristic is $y$ rather than $p$ so $f_{d}^{v}(\boldsymbol{a x}+\boldsymbol{b} \boldsymbol{y}) \in \mathbb{Z}((y))$.

In Section 4, at the level of $\mathbb{C}$-valued points, we expressed $\rho_{d}^{-1}(\boldsymbol{a} \boldsymbol{x}+\boldsymbol{b} \boldsymbol{y})$ as a disjoint union of closed subsets $\operatorname{Hilb}^{\bullet}\left(X, C_{x, y, \lambda, \mu}\right)$. We obtain

$$
f_{d}^{v}(\boldsymbol{a} \boldsymbol{x}+\boldsymbol{b} \boldsymbol{y})=\sum_{\lambda \vdash \boldsymbol{a}} \sum_{\boldsymbol{\mu} \vdash \boldsymbol{b}} y^{\chi\left(\mathcal{O}_{\left.C_{x, y, \lambda, \mu}\right)+n}\right.} \int_{\operatorname{Hilb}^{n}\left(X, C_{x, y, \lambda, \mu}\right)} v d e,
$$

where $v$ is the Behrend function of $\operatorname{Hilb}^{\bullet \bullet \bullet}(X)$ and $C_{x, y, \lambda, \mu}$ denotes the following Cohen-Macaulay curve

$$
C_{\boldsymbol{x}, \boldsymbol{y}, \lambda, \boldsymbol{\mu}}:=B \cup_{i}\left(\lambda^{(i)} F_{x_{i}}\right) \cup_{j}\left(\mu^{(j)} F_{y_{j}}\right) .
$$

Recall that the factor $y^{\chi\left(\mathcal{O}_{\left.C_{x, y, \lambda, \mu}\right)}\right)}$ comes from the fact that $\operatorname{Hilb}^{\bullet \bullet}(X)$ is indexed by $\chi\left(\mathcal{O}_{Z}\right)$ and $\operatorname{Hilb}^{\bullet}\left(X, C_{x, y, \lambda, \mu}\right)$ by the length of $I_{C_{x, y, \lambda, \mu}} / I_{Z}$ (Definition 4$)$. We apply Conjecture 21 to the above and also use

$$
v\left(\left[C_{x, y, \lambda, \mu}\right]\right)=(-1)^{\chi\left(\mathcal{O}_{S}\right)-\chi\left(\mathcal{O}_{C_{x, y, \lambda, \mu}}\right)}
$$


the highly nontrivial result given in Corollary 26 and proved in the next section. We find

$$
\begin{aligned}
f_{d}^{v}(\boldsymbol{a x}+\boldsymbol{b} \boldsymbol{y}) & =\sum_{\lambda \vdash \boldsymbol{a}} \sum_{\boldsymbol{\mu} \vdash \boldsymbol{b}} y^{\chi\left(\mathcal{O}_{C_{x, y, \lambda, \mu}}\right)} \sum_{n=0}^{\infty} y^{n}(-1)^{n} v\left(\left[C_{\boldsymbol{x}, \boldsymbol{y}, \lambda, \boldsymbol{\mu}}\right]\right) \int_{\mathrm{Hilb}^{n}\left(X, C_{\boldsymbol{x}, \boldsymbol{y}, \lambda, \boldsymbol{\mu}}\right)} d e \\
& =(-1)^{\chi\left(\mathcal{O}_{S}\right)} \sum_{\lambda \vdash \boldsymbol{a}} \sum_{\boldsymbol{\mu} \vdash \boldsymbol{b}} \sum_{n=0}^{\infty}(-y)^{\chi\left(\mathcal{O}_{\left.C_{x, y, \lambda, \mu}\right)+n}\right.} \int_{\mathrm{Hib}^{n}\left(X, C_{x, y, \lambda, \mu}\right)} d e .
\end{aligned}
$$

After the substitution $-y=p$, we find that the above is equivalent to

$$
f_{d}^{v}(\boldsymbol{a} \boldsymbol{x}+\boldsymbol{b} \boldsymbol{y})=(-1)^{\chi\left(\mathcal{O}_{s}\right)} f_{d}(\boldsymbol{a} \boldsymbol{x}+\boldsymbol{b} \boldsymbol{y}) .
$$

It follows that

$$
\mathrm{DT}(X)=(-1)^{\chi\left(\mathcal{O}_{s}\right)} \widehat{\mathrm{DT}}(X)
$$

after the substitution $p=-y$ as asserted by Theorem 3 .

The case of $\mathrm{DT}_{\text {fib }}(X)$ (previously shown by Toda) proceeds similarly except that it does not require the difficult deformation result of the next section. Indeed, in this case, we only need to know that the value of the Behrend function at a partition thickened fiber curve is 1

$$
v\left(\left[\cup_{i}\left(\lambda^{(i)} F_{x_{i}}\right)\right]\right)=1 .
$$

This follows from the fact that subschemes in $X$ of the form $p^{-1}(Z)$, where $Z$ is a zero-dimensional subscheme of $T$, form a component of $\operatorname{Hilb}(X)$ which is isomorphic to the Hilbert scheme of points on $T$ and hence smooth and even dimensional. While this can be proven directly, one can also do a similar (but easier) computation as we do in the proof of Theorem 24 in Section 9.

\section{Smoothness and infinitesimal deformations}

In this section we show that the locus of partition thickened comb curves lies in the nonsingular locus of $\operatorname{Hilb}(X)$ and we compute the dimension of $\operatorname{Hilb}(X)$ at those points. As a corollary, we determine the value of the Behrend function at the points of the Hilbert scheme corresponding to partition thickened comb curves. This is the key technical result required in Section 8 to promote our computation of $\widehat{\mathrm{DT}}(X)$ to a computation of the Behrend function version $\mathrm{DT}(X)$.

We begin by stating the three main results of this section.

THEOREM 23. Let $B \subset T$ be a smooth curve contained in a smooth surface $T$. Define $V_{l} \subset \operatorname{Hilb}^{d}(T)$ to be the locus of points parameterizing zero-dimensional subschemes $Z \subset T$ of length $d$ such that the length of the scheme-theoretic intersection $Z \cap B$ is $l$. Then $V_{l}$ is locally closed and smooth of dimension $2 d-l$. 
THEOREM 24. Let $\lambda^{(1)}, \ldots, \lambda^{(n)}$ be partitions and let $C=B \cup_{i}\left(\lambda^{(i)} F_{x_{i}}\right)$ be a partition thickened comb curve. The Zariski tangent space of $\mathrm{Hilb}(X)$ at the point $[C]$, which is given by $\operatorname{Hom}\left(I_{C}, \mathcal{O}_{C}\right) \cong \operatorname{Ext}_{0}^{1}\left(I_{C}, I_{C}\right)$, has dimension

$$
h^{0}\left(N_{B / T}\right)+\sum_{i=1}^{n}\left(2\left|\lambda^{(i)}\right|-\lambda_{1}^{(i)}\right) .
$$

THEOREM 25. The locus of partition thickened comb curves is contained in the nonsingular locus of $\mathrm{Hilb}(X)$.

COROLlary 26. The value of the Behrend function at $[C] \in \operatorname{Hilb}(X)$ for a partition thickened comb curve $C=B \cup_{i}\left(\lambda^{(i)} F_{x_{i}}\right)$ is given by

$$
v([C])=(-1)^{\chi\left(\mathcal{O}_{S}\right)-\chi\left(\mathcal{O}_{C}\right)} .
$$

Proof. By [2], the Behrend function on a smooth scheme $V$ is $(-1)^{\operatorname{dim} V}$ and so by Theorems 25 and 24

$$
v([C])=(-1)^{h^{0}\left(N_{B / X}\right)} \prod_{i=1}^{n}(-1)^{\lambda_{1}^{(i)}} .
$$

Lemma 30 and Lemma 11 say that

$$
h^{0}\left(N_{B / X}\right)=\chi\left(\mathcal{O}_{S}\right)-\chi\left(\mathcal{O}_{B}\right), \quad \chi\left(\mathcal{O}_{C}\right)=\chi\left(\mathcal{O}_{B}\right)-\sum_{i} \lambda_{1}^{(i)}
$$

which, when substituted into the above, prove the corollary.

The most difficult of the above results is Theorem 24 and its proof occupies the majority of this section.

Our method for computing the dimension of deformation spaces is an adaption of Haiman's method for computing infinitesimal deformations of zero-dimensional subschemes on a surface [9]. Indeed, the proof of Theorem 23 follows directly using Haiman's argument. For Theorem 24, we use Haiman's method to study local deformations of $C$ in the formal neighborhoods of the points $x_{i}$, but we use the global geometry to keep track of which local deformations extend.

9.1. Setup for the proof of Theorem 24. For notational simplicity we first treat the case where there is a single partition thickened fiber $F=F_{x}$ at $x \in B$, 
that is

$$
C=B \cup \lambda F
$$

where $\left(\lambda_{1} \geqslant \lambda_{2} \geqslant \cdots \geqslant \lambda_{l}\right)$ is an integer partition of length $l$.

Consider the divisors

$$
S, \quad R=\operatorname{Tot}\left(\left.K_{S}\right|_{F}\right), \quad T=\operatorname{Tot}\left(\left.K_{S}\right|_{B}\right)
$$

and let $(r, s, t)$ be formal local coordinates at $x$ such that

$$
R=\{r=0\}, \quad S=\{s=0\}, \quad T=\{t=0\} .
$$

The formal local ring $\widehat{\mathcal{O}}_{X, x} \cong \mathbb{C}[[r, s, t]]$ has a basis as a $\mathbb{C}$-vector space given by monomials $\left\{r^{\rho} s^{\sigma} t^{\tau}\right\}$ for $(\rho, \sigma, \tau) \in\left(\mathbb{Z}_{\geqslant 0}\right)^{3}$. We visualize these basis vectors as unit cubes in the positive octant of $\mathbb{R}^{3}$ with the monomial $r^{\rho} s^{\sigma} t^{\tau}$ corresponding to the cube whose corner closest to the origin is at $(\rho, \sigma, \tau)$.

9.2. Exact sequences. The ideal sheaf $I_{C}$ has a locally free resolution of the form

$$
0 \rightarrow \oplus_{\beta} R_{\beta} \rightarrow \oplus_{\alpha} G_{\alpha} \rightarrow I_{C} \rightarrow 0
$$

where $G_{\alpha}$ (the "generators") and $R_{\beta}$ (the "relations") are of the form

$$
\mathcal{O}(-\rho R-\sigma S-\tau T) .
$$

Indeed, we can explicitly take the collection of $(\rho, \sigma, \tau)$ for $G_{\alpha}$ to be

$$
\left\{\left(\lambda_{1}, 0,1\right),\left(\lambda_{2}, 1,0\right),\left(\lambda_{3}, 2,0\right), \ldots,\left(\lambda_{l}, l-1,0\right),(0, l, 0)\right\} .
$$

Note that the $\tau$ component is 1 for the first generator, and zero for all others.

We also have the sequence

$$
0 \rightarrow \mathcal{O}_{C} \rightarrow \mathcal{O}_{B} \oplus \mathcal{O}_{\lambda F} \rightarrow \mathcal{O}_{\lambda_{1} x} \rightarrow 0
$$

where $\lambda_{1} x=B \cap \lambda F$ is the length $\lambda_{1}$ subscheme of $B$ supported at $x$.

By standard homological algebra, we have that $\operatorname{Hom}\left(I_{C}, \mathcal{O}_{C}\right)$ is $H^{0}$ of the complex

$$
\operatorname{Hom}\left(\left[\oplus_{\beta} R_{\beta} \rightarrow \oplus_{\alpha} G_{\alpha}\right],\left[\mathcal{O}_{B} \oplus \mathcal{O}_{\lambda F} \rightarrow \mathcal{O}_{\lambda_{1} x}\right]\right)
$$

Namely, we have that $\operatorname{Hom}\left(I_{C}, \mathcal{O}_{C}\right)$ is given by the kernel of the map

$\operatorname{Hom}\left(\oplus_{\alpha} G_{\alpha}, \mathcal{O}_{B} \oplus \mathcal{O}_{\lambda F}\right) \stackrel{\Phi_{1} \oplus \Phi_{2}}{\longrightarrow} \operatorname{Hom}\left(\oplus_{\alpha} G_{\alpha}, \mathcal{O}_{\lambda_{1} x}\right) \oplus \operatorname{Hom}\left(\oplus_{\beta} R_{\beta}, \mathcal{O}_{B} \oplus \mathcal{O}_{\lambda F}\right)$

This identification of $\operatorname{Hom}\left(I_{C}, \mathcal{O}_{C}\right)$ has a straightforward interpretation: a homomorphism $I_{C} \rightarrow \mathcal{O}_{C}$ is determined by what it is on each of the generators 


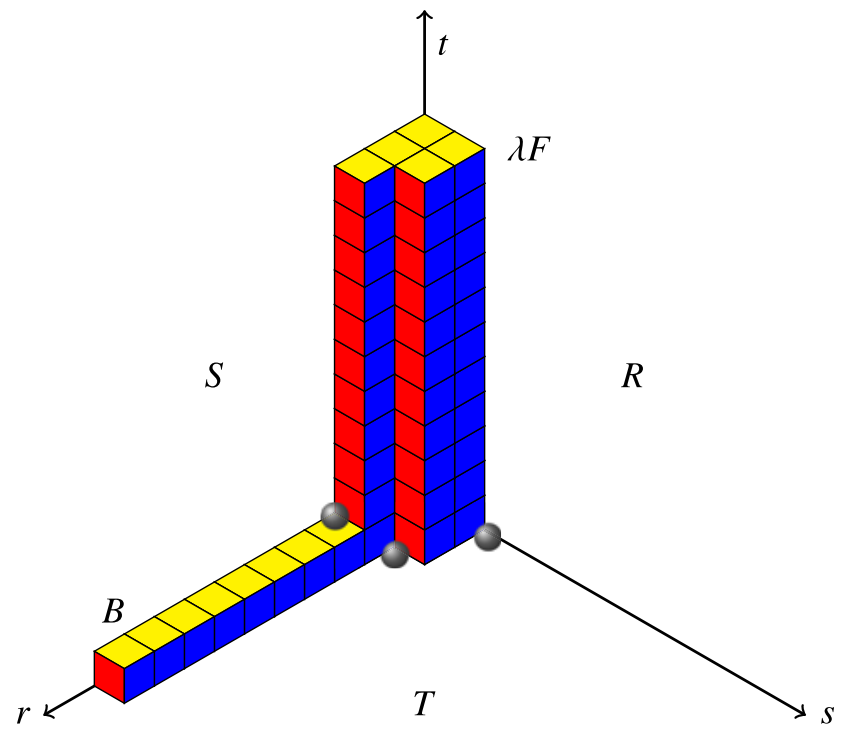

Figure 2. Monomials in the local ring $\widehat{\mathcal{O}}_{X, x} \cong \mathbb{C}[[r, s, t]]$ are represented by cubes. Cubes shown are the monomials in $\widehat{\mathcal{O}}_{C, x}$. The gray balls are located at monomials which generate $\widehat{I}_{C, x}$.

of $I_{C}$, considered as maps to $\mathcal{O}_{B}$ and to $\mathcal{O}_{\lambda F}$. To be in the kernel of $\Phi_{1}$ just means that these maps should agree on $B \cap \lambda F$ and to be in the kernel of $\Phi_{2}$ means that the images must obey the module relations. We will make this combinatorially more explicit by studying the restriction of the homomorphisms $\oplus_{\alpha} G_{\alpha} \rightarrow \mathcal{O}_{B} \oplus \mathcal{O}_{\lambda F}$ to $\widehat{X}_{x} \cong \operatorname{Spec} \mathbb{C}[[r, s, t]]$.

9.3. Combinatorics of Haiman arrows. When restricted to the local ring $\widehat{\mathcal{O}}_{X, x} \cong \mathbb{C}[[r, s, t]], \mathcal{O}_{C}$ is spanned over $\mathbb{C}$ by the monomials $r^{\rho} s^{\sigma} t^{\tau}$, where $(\rho, \sigma, \tau)$ are of the form $(\rho, 0,0)$ or $(\rho, \sigma, \tau)_{(\rho, \sigma) \in \lambda}$ and $I_{C}$ is spanned by the complementary monomials. As previously discussed, we view these monomials as cubes in the positive octant, see Figure 2.

We call the cubes corresponding to $(\rho, 0,0)$ and $(\rho, \sigma, \tau)_{(\rho, \sigma) \in \lambda}$ the $B$-cubes and $\lambda F$-cubes respectively and the cubes in the union are called $C$-cubes. The complements of the $C$-cubes are the $I_{C}$-cubes.

\section{A Haiman arrow}

$$
(\rho, \sigma, \tau) \rightarrow\left(\rho^{\prime}, \sigma^{\prime}, \tau^{\prime}\right)
$$


is a vector whose tail $(\rho, \sigma, \tau)$ is an $I_{C}$-cube and whose head $\left(\rho^{\prime}, \sigma^{\prime}, \tau^{\prime}\right)$ is a $C$ cube.

The Haiman arrows form a basis for the $\mathbb{C}$-linear maps from $\widehat{I}_{C, x}$ to $\widehat{\mathcal{O}}_{C, x}$. We wish to determine a basis for $\operatorname{Hom}\left(I_{C}, \mathcal{O}_{C}\right)$ in terms of Haiman arrows.

The generators of $I_{C}$ correspond to the cubes in the corners of the set of $I_{C^{-}}$ cubes. They are located at $(\rho, \sigma, \tau)$ where $(\rho, \sigma)$ are the corners just outside of $\lambda$ and $\tau=0$ unless $\sigma=0$ in which case $\tau=1$ (they are indicated by the gray balls in Figure 2). A generator at $(\rho, \sigma, \tau)$ corresponds to the image of $G_{\alpha} \rightarrow \mathcal{O}$ where $G_{\alpha} \cong \mathcal{O}(-\rho R-\sigma S-\tau T)$. The summands of

$$
\operatorname{Hom}\left(\oplus_{\alpha} G_{\alpha}, \mathcal{O}_{B} \oplus \mathcal{O}_{\lambda F}\right) \cong \oplus_{\alpha} H^{0}\left(B, G_{\alpha}^{\vee} \otimes \mathcal{O}_{B}\right) \oplus H^{0}\left(F, G_{\alpha}^{\vee} \otimes \mathcal{O}_{\lambda F}\right)
$$

are interpreted as follows. For $G_{\alpha} \cong \mathcal{O}(-\rho R-\sigma S-\tau T)$, a homomorphism in $\operatorname{Hom}\left(G_{\alpha}, \mathcal{O}_{B}\right)$ or $\operatorname{Hom}\left(G_{\alpha}, \mathcal{O}_{\lambda F}\right)$ is determined by a linear combination of Haiman arrows from $(\rho, \sigma, \tau)$ to (respectively) some $B$-cube or $\lambda F$-cube $\left(\rho^{\prime}\right.$, $\left.\sigma^{\prime}, \tau^{\prime}\right)$. The location of the head of such a Haiman arrow is determined by the order of vanishing of the corresponding section of $H^{0}\left(B, G_{\alpha}^{\vee} \otimes \mathcal{O}_{B}\right)$ or $H^{0}(F$, $\left.G_{\alpha}^{\vee} \otimes \mathcal{O}_{\lambda F}\right)$-the head will occur at $\left(\rho^{\prime}, \sigma^{\prime}, \tau^{\prime}\right)$ if the corresponding section is order $r^{\rho^{\prime}} s^{\sigma^{\prime}} t^{\tau^{\prime}}$.

We wish to determine a basis for $\operatorname{Hom}\left(I_{C}, \mathcal{O}_{C}\right) \cong \operatorname{Ker}\left(\Phi_{1} \oplus \Phi_{2}\right)$ in terms of Haiman arrows. To be in the kernel of $\Phi_{1}$ just means that a Haiman arrow whose head is both a $B$-cube and a $\lambda F$-cube must arise as sections of both $H^{0}\left(B, G_{\alpha}^{\vee} \otimes\right.$ $\left.\mathcal{O}_{B}\right)$ and $H^{0}\left(F, G_{\alpha}^{\vee} \otimes \mathcal{O}_{\lambda F}\right)$. As for the kernel of $\Phi_{2}$, the key observation is the following, essentially due to Haiman [9]:

REMARK 27. The equations defining the kernel of $\Phi_{2}$ equate two Haiman arrows which are obtained from one another by translation through other Haiman arrows. Moreover, if a Haiman arrow can be translated so that its head passes into an octant with a negative coordinate (without its tail ever leaving the $I_{C}$-cubes) then it must be zero.

We now analyze the possible equivalence classes of Haiman arrows.

9.4. Haiman arrows to $\lambda \boldsymbol{F}$-cubes. Let $G_{\alpha} \cong \mathcal{O}(-\rho R-\sigma S-\tau T)$ be a generating line bundle and consider the sections $H^{0}\left(F, G_{\alpha}^{\vee} \otimes \mathcal{O}_{\lambda F}\right)$. A basis for this vector space corresponds to the possible Haiman arrows $(\rho, \sigma, \tau) \rightarrow\left(\rho^{\prime}, \sigma^{\prime}\right.$, $\left.\tau^{\prime}\right)$ to $\lambda F$-cubes. Since the normal bundle of $F$ in $X$ is trivial, $\mathcal{O}(R)$ and $\mathcal{O}(S)$ are trivial restricted to $F$. Thus

$$
G_{\alpha}^{\vee} \otimes \mathcal{O}_{\lambda F} \cong \mathcal{O}_{\lambda F}(\rho R+\sigma S+\tau T) \cong \mathcal{O}_{\lambda F}(\tau x)
$$




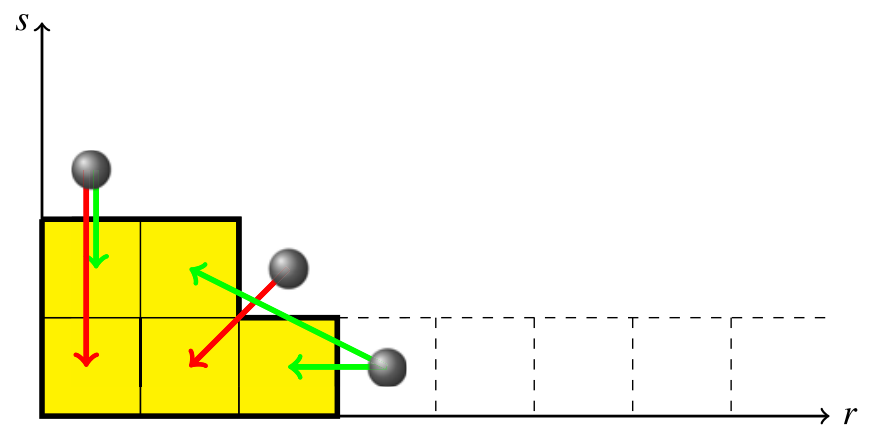

Figure 3. Examples of Haiman arrows to $\lambda F$-cubes. The green arrows are nonzero and the red arrows are necessarily zero.

Since $\tau$ is either 0 or 1 for the generators $G_{\alpha}$, the Haiman arrows correspond to

$$
H^{0}\left(F, \mathcal{O}_{\lambda F}\right) \quad \text { if } \tau=0, \quad H^{0}\left(F, \mathcal{O}_{\lambda F}(x)\right) \quad \text { if } \tau=1 .
$$

In both cases, this space has a basis of sections which in the local coordinates are given by $\left\{r^{\rho^{\prime}} s^{\sigma^{\prime}} t^{\tau}\right\}_{\left(\rho^{\prime}, \sigma^{\prime}\right) \in \lambda}$. Note that the sections we consider above are uniquely determined by their value on the formal neighborhood $\widehat{X}_{x} \cong \operatorname{Spec} \mathbb{C}[[r, s, t]]$, a property which uses crucially the fact that the genus of $F$ is 1 .

We have seen that the possible Haiman arrows to $\lambda F$-cubes are given by

$$
(\rho, \sigma, \tau) \rightarrow\left(\rho^{\prime}, \sigma^{\prime}, \tau^{\prime}\right)
$$

where $(\rho, \sigma, \tau)$ is a generating cube, $\tau^{\prime}=\tau$ and $\left(\rho^{\prime}, \sigma^{\prime}\right) \in \lambda$. In particular, the direction of the arrows is horizontal since there is no $\tau$ component in $\left(\rho-\rho^{\prime}\right.$, $\left.\sigma-\sigma^{\prime}, 0\right)$.

Since all the Haiman arrows to $\lambda F$-cubes are horizontal, we view them from above in the $(r, s)$ plane (see Figure 3 ).

If the direction of the Haiman arrow is strictly southwest (that is, it has strictly negative $\rho$ and $\sigma$ components), then by translating (see Remark 27) along the contour of $\lambda$ to the edge of the $s$-axis, the arrow can be equated to an arrow whose head has a negative $\rho$ component and is thus zero. There are no strictly northeast pointing Haiman arrows, so all nonzero Haiman arrows must be weakly northwest pointing or weakly southeast pointing. Translating a weakly northwest pointing arrow as far to the northwest as possible, we find that its head will either cross the $s$-axis (and hence be 0 ) or it will be at the top of a column of $\lambda$ and its tail just outside a row. Indeed, for each square in $\lambda$, there is exactly one equivalence class of weakly northwest pointing Haiman arrows represented by the arrow going from 
just outside the box's row to the top of the box's column. Similarly, there is one equivalence class of weakly southeast pointing Haiman arrows for each box in $\lambda$ represented by the arrow going from just outside the top of the box's column to the end of the box's row.

The above accounts for precisely $2|\lambda|$ different equivalence classes of Haiman arrows to $\lambda F$-cubes. However, $\lambda_{1}$ of these arrows have their head in a $B$-cube, namely the southeast pointing arrows whose tails are just above the top of $\lambda$ and whose head is the last square in the first row of $\lambda$. Note that the northwest pointing Haiman arrows whose heads are in the first row of $\lambda$ are necessarily strictly west pointing and hence originate at the generator whose $\tau$ component is 1 . Therefore the heads of these arrows also have $\tau$ component 1 and so they are not $B$-cubes.

We thus have exactly $2|\lambda|-\lambda_{1}$ distinct equivalence classes of Haiman arrows to $\lambda F$-cubes which are not also arrows to $B$-cubes.

9.5. Haiman arrows to $\boldsymbol{B}$-cubes. Any nonzero Haiman arrow to a $B$-cube must have a tail with coordinates $(\rho, 0,1)$ or $(\rho, 1,0)$ since if not, it could be translated (see Remark 27) to an arrow whose head has negative $\tau$ or $\sigma$ coordinates by first translating sufficiently far in the positive $\rho$-direction and then translating the tail so that it is just outside of the $B$-cubes. A Haiman arrow to a $B$-cube whose tail is $(\rho, 0,1)$ or $(\rho, 1,0)$ corresponds respectively to a section in $H^{0}\left(B, \mathcal{O}_{B}(\rho R+T)\right)$ or $H^{0}\left(B, \mathcal{O}_{B}(\rho R+S)\right)$. Since

$$
\mathcal{O}_{B}(R) \cong \mathcal{O}_{B}(x), \quad \mathcal{O}_{B}(T) \cong N_{B / S}, \quad \mathcal{O}_{B}(S) \cong N_{B / T},
$$

we see that the Haiman arrows from $(\rho, 0,1)$ or $(\rho, 1,0)$ to $B$-cubes are given by

$$
H^{0}\left(B, N_{B / S}(\rho x)\right) \quad \text { or } \quad H^{0}\left(B, N_{B / T}(\rho x)\right)
$$

respectively. The head of such a Haiman arrow is $\left(\rho^{\prime}, 0,0\right)$ where $\rho^{\prime}$ is the order of vanishing at $x$ of the corresponding section.

LEMma 28. Let $(\rho, 0,1) \rightarrow\left(\rho^{\prime}, 0,0\right)$ or $(\rho, 1,0) \rightarrow\left(\rho^{\prime}, 0,0\right)$ be a nonzero Haiman arrow. Then $\rho^{\prime} \geqslant \rho$.

Proof. Consider a Haiman arrow $(\rho, 0,1) \rightarrow\left(\rho^{\prime}, 0,0\right)$ with $\rho^{\prime}<\rho$. Then this arrow can be translated so that its head is a $\lambda F$-cube; however, we saw in the previous subsection that Haiman arrows to $\lambda F$-cubes must be horizontal and so this must be zero. Consider next a Haiman arrow $(\rho, 1,0) \rightarrow\left(\rho^{\prime}, 0,0\right)$ with $\rho^{\prime}<$ $\rho$. Then this arrow may be translated so that it is a strictly southwest pointing Haiman arrow to a $\lambda F$-cube which we showed in the previous subsection must be zero. (Are you still with us dear reader? We are deep in the weeds now, but are almost done.) 
By the lemma, we conclude that the only sections of $H^{0}\left(B, N_{B / S}(\rho x)\right)$ or $H^{0}\left(B, N_{B / T}(\rho x)\right)$ which correspond to nonzero Haiman arrows vanish to order at least $\rho$ at $x$, and thus they are necessarily in the image of the maps

$$
\begin{aligned}
& H^{0}\left(B, N_{B / S}\right) \rightarrow H^{0}\left(B, N_{B / S}(\rho x)\right), \\
& H^{0}\left(B, N_{B / T}\right) \rightarrow H^{0}\left(B, N_{B / T}(\rho x)\right) .
\end{aligned}
$$

By Lemma $30, H^{0}\left(B, N_{B / S}\right)=0$. On the other hand, $H^{0}\left(B, N_{B / T}\right)$ can be nonzero and these deformations do occur, they correspond to global deformations of $B$ in the $K_{S}$-direction.

In conclusion, we have completely classified all possible Haiman arrows up to equivalence and have thus constructed an explicit basis for

$$
\operatorname{Hom}\left(I_{C}, \mathcal{O}_{C}\right) \cong \operatorname{Ker}\left(\Phi_{1} \oplus \Phi_{2}\right)
$$

They consist of the $2|\lambda|-\lambda_{1}$ Haiman arrows to $\lambda F$-cubes which do not go to $B$ cubes and the $h^{0}\left(B, N_{B / T}\right)=h^{0}\left(B, N_{B / X}\right)$ dimensional space of Haiman arrows going to $B$-cubes. We have thus proved that

$$
\operatorname{dim} \operatorname{Hom}\left(I_{C}, \mathcal{O}_{C}\right)=h^{0}\left(B, N_{B / T}\right)+2|\lambda|-\lambda_{1}
$$

for $C=B \cup \lambda F$. Our argument extends essentially word for word to the case where $C=B \cup_{i}\left(\lambda^{(i)} F_{x_{i}}\right)$ has several partition thickened fibers. Whether the fiber is smooth or nodal plays no role. We have thus proved Theorem 24.

9.6. Proof of Theorem 25. Let $C=B \cup_{i=1}^{n}\left(\lambda^{(i)} F_{x_{i}}\right)$ be a partition thickened comb curve and let

$$
d=\sum_{i=1}^{n}\left|\lambda^{(i)}\right|, \quad l=\sum_{i=1}^{n} \lambda_{1}^{(i)} .
$$

To prove Theorem 25 it will suffice to construct a flat family of distinct subschemes of $X$, containing $C$ as a member, and over a base $W$ which is smooth and of dimension

$$
h^{0}\left(N_{B / T}\right)+2 d-l .
$$

Indeed, Theorem 24 then implies that the induced injective map $W \rightarrow \operatorname{Hilb}(X)$ is a local isomorphism and the assertion of Theorem 25 follows.

Let

$$
H^{0}:=H^{0}\left(B, N_{B / T}\right)
$$

and let

$$
V_{l} \subset \operatorname{Hilb}^{d}(T)
$$


be the stratum defined in Theorem 23. Let $W=H^{0} \times V_{l}$ so by Theorem $23, W$ is smooth and of dimension $h^{0}\left(N_{B / T}\right)+2 d-l$. We wish to construct a family over $W$ of distinct subschemes.

Since $T=\operatorname{Tot}\left(N_{B / T}\right)$, given $\theta \in H^{0}$, we get an automorphism of $T$, which we call $\Theta$, given by

$$
\Theta:(p, v) \mapsto(p, v+\theta(p)),
$$

where $p \in B$ and $\left.v \in T\right|_{p}$.

We will construct a family of subschemes of $X$, flat over the base $H^{0} \times V_{l}$, which over a point $(\theta, Z) \in H^{0} \times V_{l}$ is the subscheme

$$
C_{\theta}=\Theta(B) \cup p^{-1}(\Theta(Z)) .
$$

Clearly, all such subschemes are distinct, and moreover, every partition thickened comb curve is of the above form (with $\theta=0$ so that $\Theta=$ id).

Formally, we construct the universal subscheme

$$
\mathcal{C} \subset H^{0} \times V_{l} \times X
$$

flat over $H^{0} \times V_{l}$ as follows. Consider the diagram

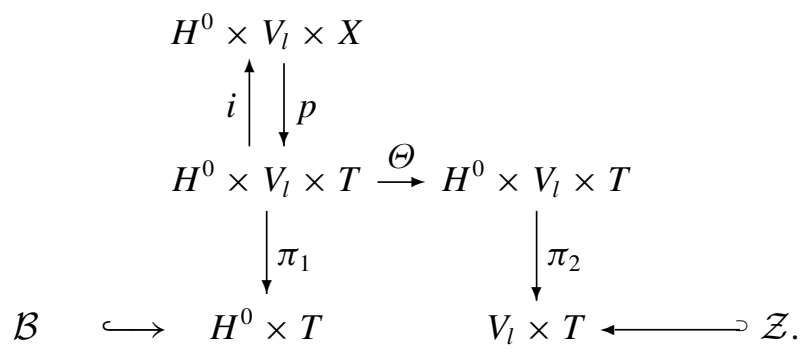

In the above diagram, $\mathcal{Z} \subset V_{l} \times T$ is the family of subschemes of $T$ induced by the universal subscheme over $\operatorname{Hilb}^{d}(T), \mathcal{B} \subset H^{0} \times T$ is the family of curves in $T$ given by $H^{0}$, explicitly $\mathcal{B}$ is given by the set of points $(\theta, p, \theta(p))$. The maps $\pi_{1}$ and $\pi_{2}$ are the obvious projections and the maps $p$ and $i$ are the projection and the zero section of the elliptic fibration $X \rightarrow T$. We are also adopting the general abuse of notation that we drop factors of the identity map from the notation, that is, if $f: A \rightarrow B$ we denote also by $f$ the map $f \times \operatorname{id}_{C}: A \times C \rightarrow B \times C$.

Then the subscheme

$$
\mathcal{C}=i\left(\pi_{1}^{-1}(\mathcal{B})\right) \cup\left(p \circ \Theta \circ \pi_{2}\right)^{-1}(\mathcal{Z}) \subset H^{0} \times V_{l} \times X
$$

is the desired universal subscheme over $H^{0} \times V_{l}$. 
9.7. Proof of Theorem 23. The constructible function $\operatorname{Hilb}^{d}(T) \rightarrow \mathbb{Z}$ given by

$$
Z \mapsto \operatorname{leng}(Z \cap B)
$$

is upper semicontinuous and thus $V_{l}$ is locally closed.

There is a dense open set on $V_{l}$ isomorphic to

$$
\operatorname{Sym}^{l}(B) \times \operatorname{Hilb}^{d-l}(T-B),
$$

which is clearly smooth and of dimension $2 d-l$. Therefore, to prove the theorem it suffices to show that

$$
\operatorname{dim} T_{[Z]} V_{l}=2 d-l,
$$

where $Z \subset T$ is a subscheme which is set-theoretically (but not necessarily scheme-theoretically) supported on $B$. Moreover, we can easily reduce to the case where $Z$ is supported at a single point $p \in B$. By choosing formal local coordinates $(r, s)$ on $T$ at $p$ such that $B=\{s=0\}$, we are reduced to considering the case

$$
T=\operatorname{Spec} \mathbb{C}[r, s], \quad B=\{s=0\}, \text { and } Z \subset T \text { supported at } 0 .
$$

Finally, since $\left(\mathbb{C}^{*}\right)^{2}$ acts on $V_{l}$ in this case, it suffices to compute $\operatorname{dim} T_{[Z]} V_{l}$ at $\left(\mathbb{C}^{*}\right)^{2}$-fixed subschemes $Z \subset T$. Recall that the fixed subschemes are given by $Z_{\lambda}$ (see Section 3) defined by monomial ideals $I_{\lambda} \subset \mathbb{C}[r, s]$ corresponding to partitions $\lambda$ of $d$ which in this case have $\lambda_{1}=l$, because leng $\left(Z_{\lambda} \cap B\right)=l$.

Therefore, we need only to prove the following lemma:

LEMMA 29. Let $\lambda=\left(\lambda_{1} \geqslant \cdots \geqslant \lambda_{k}\right)$ be a partition of $d$ with $\lambda_{1}=$ l. Let $Z_{\lambda} \subset$ $\mathbb{C}^{2}=\operatorname{Spec} \mathbb{C}[r, s]$ be defined by the monomial ideal

$$
I_{\lambda}=\left(r^{\lambda_{1}}, r^{\lambda_{2}} s, \ldots, r^{\lambda_{k}} s^{k-1}, s^{k}\right) .
$$

Let $V_{l} \subset \operatorname{Hilb}^{d}\left(\mathbb{C}^{2}\right)$ be as in Theorem 23. Then

$$
\operatorname{dim} T_{\left[Z_{\lambda}\right]} V_{l}=2 d-l .
$$

Proof. The tangent space

$$
T_{\left[Z_{\lambda}\right]} V_{l} \subset T_{\left[Z_{\lambda}\right]} \operatorname{Hilb}^{d}\left(\mathbb{C}^{2}\right)
$$

is cut out by the equations obtained by linearizing the condition

$$
\operatorname{leng}(Z \cap\{s=0\})=l
$$

at $Z_{\lambda}$. In [9], Haiman has given a very explicit basis for $T_{Z_{\lambda}} \operatorname{Hilb}^{d}\left(\mathbb{C}^{2}\right)$ in terms of what we called Haiman arrows in Section 9.3. Namely, consider all Haiman arrows $(\rho, \sigma) \rightarrow\left(\rho^{\prime}, \sigma^{\prime}\right)$ of one of the following two forms: 
(1) $(\rho, \sigma) \rightarrow\left(\rho^{\prime}, \sigma^{\prime}\right)$ is a southeast pointing arrow with $(\rho, \sigma)$ located at a box just above the top of a column of $\lambda$ and $\left(\rho^{\prime}, \sigma^{\prime}\right)$ located at a box which is the furthest to the right in a row of $\lambda$.

(2) $(\rho, \sigma) \rightarrow\left(\rho^{\prime}, \sigma^{\prime}\right)$ is a northwest pointing arrow with $(\rho, \sigma)$ located at a box just to the right of a row of $\lambda$ and $\left(\rho^{\prime}, \sigma^{\prime}\right)$ located at a box which is at the top of a column of $\lambda$.

There are $2 d$ such arrows, $d$ of each kind. The infinitesimal deformation corresponding to an arrow $(\rho, \sigma) \rightarrow\left(\rho^{\prime}, \sigma^{\prime}\right)$ is given by deforming the element $r^{\rho} s^{\sigma} \in I_{\lambda}$ to

$$
r^{\rho} s^{\sigma}+\epsilon r^{\rho^{\prime}} s^{\sigma^{\prime}}
$$

where $\epsilon^{2}=0$.

For each $\phi:(\rho, \sigma) \rightarrow\left(\rho^{\prime}, \sigma^{\prime}\right)$ let $I_{Z_{\lambda}}(\phi)$ be the corresponding deformed ideal and consider

$$
\operatorname{dim}\left(\frac{\mathbb{C}[r, s]}{I_{Z_{\lambda}}(\phi)+(s)}\right) .
$$

If $\sigma^{\prime}>0$ then $I_{Z_{\lambda}}(\phi)+(s)=I_{Z_{\lambda}}+(s)$ : Haiman arrows $(\rho, \sigma) \rightarrow\left(\rho^{\prime}, \sigma^{\prime}\right)$ with $\sigma^{\prime}>0$ preserve the condition leng $(Z \cap\{s=0\})=l$ and hence lie in $T_{\left[Z_{\lambda}\right]} V_{l}$.

If $\sigma^{\prime}=0$ there are two possibilities:

(1) $(\rho, \sigma)$ is just above a column of $\lambda$ and $\left(\rho^{\prime}, \sigma^{\prime}\right)=\left(\lambda_{1}-1,0\right)$; or

(2) $\phi$ is of the form $\left(\lambda_{1}, 0\right) \rightarrow\left(\rho^{\prime}, 0\right)$ for $0 \leqslant \rho^{\prime}<\lambda_{1}$.

In Case 2, we have

$$
\frac{\mathbb{C}[r, s]}{I_{Z_{\lambda}}(\phi)+(s)} \cong \frac{\mathbb{C}[r]}{\left(r^{\lambda_{1}}+\epsilon r^{\rho^{\prime}}\right)},
$$

which has dimension $\lambda_{1}=l$ for all values of $\epsilon$ since $r^{\lambda_{1}}+\epsilon r^{\rho^{\prime}}$ has degree $\lambda_{1}$ for all values of $\epsilon$.

In Case 1, we have

$$
\frac{\mathbb{C}[r, s]}{I_{Z_{\lambda}}(\phi)+(s)} \cong \frac{\mathbb{C}[r]}{\left(r^{\lambda_{1}}, \epsilon r^{\lambda_{1}-1}\right)}
$$

which, for nonzero values of $\epsilon$, has dimension $\lambda_{1}-1$.

Thus we have found that $T_{\left[Z_{\lambda}\right]} V_{l}$ is spanned by all the arrows in the Haiman basis except for the $\lambda_{1}=l$ arrows given by Case 1 above and therefore $\operatorname{dim} T_{\left[Z_{\lambda}\right]} V_{l}=$ $2 d-l$. 


\section{Acknowledgements}

We would like to thank Tom Graber, Paul Johnson, Manfred Lehn, Oliver Leigh, Davesh Maulik, Georg Oberdieck, Rahul Pandharipande, Jørgen Rennemo, Balázs Szendrői, Richard Thomas, Qizheng Yin, and Benjamin Young for helpful conversations. We particularly thank the anonymous referee, whose comments led us to replace our previous approach (involving formal schemes and fpqc covers) by a much clearer stratification technique, which simply keeps track of the location of the embedded points with respect to $C_{\text {red }}$ (Section 5). This improved the paper significantly.

Part of this work was done during the semester Enumerative geometry of moduli spaces of sheaves in low dimension at CIB/EPFL, who provided support and excellent working conditions.

\section{Appendix A. Odds and Ends}

A.1. Elliptic surfaces. Let $p: S \rightarrow B$ be a nontrivial elliptic surface with section $B \subset S$. For simplicity, we assume that all singular fibers are irreducible nodal rational curves.

Let $X=\operatorname{Tot}\left(K_{S}\right)$ and let $T=\operatorname{Tot}\left(\left.K_{S}\right|_{B}\right)$, then clearly we have

$$
N_{B / X} \cong N_{B / S} \oplus N_{B / T}
$$

LEMMA 30. $h^{0}\left(N_{B / S}\right)=0$ and $h^{0}\left(N_{B / T}\right)=\chi\left(\mathcal{O}_{S}\right)-\chi\left(\mathcal{O}_{B}\right)$.

Proof. By a well-known fact about elliptic surfaces (see [8] or [15, III.1.1]),

$$
K_{S} \cong p^{*}\left(K_{B} \otimes L\right),
$$

where

$$
L^{\vee}=R^{1} p_{*} \mathcal{O}_{S} .
$$

Consequently, $c_{1}\left(K_{S}\right)^{2}=0$ and so Hirzebruch-Riemann-Roch says

$$
\chi\left(\mathcal{O}_{S}\right)=\frac{e(S)}{12}>0
$$

where positivity of $e(S)$ follows by pushing forward the Euler characteristic measure on $S$ to $B$

$$
e(S)=\int_{S} d e=\int_{B} p_{*}(1) d e=\# \text { of singular fibers. }
$$


On the other hand

$$
\begin{aligned}
\chi\left(\mathcal{O}_{S}\right) & =\chi\left(R^{\bullet} p_{*} \mathcal{O}_{S}\right) \\
& =\chi\left(\mathcal{O}_{B}\right)-\chi\left(L^{\vee}\right) \\
& =\operatorname{deg}(L) .
\end{aligned}
$$

By adjunction

$$
N_{B / S} \cong\left(\left.K_{S}^{\vee}\right|_{B}\right) \otimes K_{B} \cong L^{\vee} .
$$

Thus $\operatorname{deg}\left(N_{B / S}\right)=\operatorname{deg}\left(L^{\vee}\right)=-\chi\left(\mathcal{O}_{S}\right)<0$ and so $h^{0}\left(N_{B / S}\right)=0$.

Since

$$
N_{B / T}=\left.K_{S}\right|_{B}=K_{B} \otimes L,
$$

we see that

$$
h^{1}\left(N_{B / T}\right)=h^{1}\left(K_{B} \otimes L\right)=h^{0}\left(L^{\vee}\right)=h^{0}\left(N_{B / S}\right)=0,
$$

and therefore

$$
\begin{aligned}
h^{0}\left(N_{B / T}\right) & =\chi\left(N_{B / T}\right) \\
& =\operatorname{deg}\left(K_{B}\right)+\operatorname{deg}(L)+1-g(B) \\
& =\chi\left(\mathcal{O}_{S}\right)+g(B)-1 \\
& =\chi\left(\mathcal{O}_{S}\right)-\chi\left(\mathcal{O}_{B}\right) .
\end{aligned}
$$

By our assumption that $S$ is not a product,

$$
p^{*}: \operatorname{Pic}^{0}(B) \stackrel{\cong}{\longrightarrow} \operatorname{Pic}^{0}(S)
$$

is an isomorphism [15, VII.1.1]. For any $\beta \in H_{2}(S)$, we denote by $\operatorname{Hilb}^{\beta}(S)$ the Hilbert scheme of effective divisors on $S$ in class $\beta$.

Denote by $B \in H_{2}(S)$ the class of the section $B \subset S$ and by $F \in H_{2}(S)$ the class of the fiber. Then we have the following commutative diagram

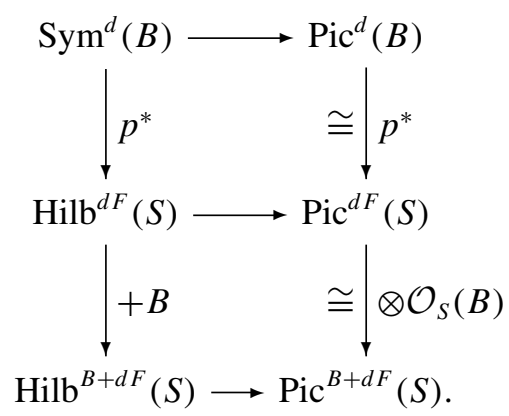

The horizontal arrows are Abel-Jacobi maps. The vertical arrows are induced by pull-back and adding the section $B \subset S$. 
LEMMA 31. The above maps induce a bijective morphism

$$
\operatorname{Sym}^{d}(B) \rightarrow \operatorname{Hilb}^{B+d F}(S) .
$$

Proof. Clearly $p^{*}$ gives an isomorphism $\operatorname{Sym}^{d}(B) \cong \operatorname{Hilb}^{d F}(S)$ and $+B$ defines a closed embedding $\operatorname{Hilb}^{d F}(S) \hookrightarrow \operatorname{Hilb}^{B+d F}(S)$. Thus it suffices to show that

$$
\operatorname{Sym}^{d}(B) \rightarrow \operatorname{Hilb}^{B+d F}(S)
$$

is surjective on closed points.

For surjectivity, suppose $D^{\prime}$ is an effective divisor with class $B+d F$ which does not lie in the image. First, we note that for any fiber $F$ we have $D^{\prime} \cdot F=1$. Therefore $D^{\prime}$ contains a section $B^{\prime} \subset S$ as an effective summand. Moreover, $B \neq B^{\prime}$ or else $D^{\prime}$ would lie in the image. Next, we take any $D$ in the image and compare $D$ and $D^{\prime}$. Then

$$
\mathcal{O}_{S}\left(D-D^{\prime}\right) \in \operatorname{Pic}^{0}(S) \cong \operatorname{Pic}^{0}(B) .
$$

Therefore after re-arranging we find that there are distinct fibers $F_{x_{i}}, F_{y_{j}}$, and $a_{i} \geqslant 0, b_{j} \geqslant 0$ such that

$$
B+\sum_{i} a_{i} F_{x_{i}} \sim_{\operatorname{lin}} B^{\prime}+\sum_{j} b_{j} F_{y_{j}}
$$

where $\sim_{\text {lin }}$ denotes linear equivalence. Hence there exists a pencil $\left\{C_{t}\right\}_{t \in \mathbb{P}^{1}}$ of effective divisors such that

$$
C_{0}=B+\sum_{i} a_{i} F_{x_{i}}, \quad C_{\infty}=B^{\prime}+\sum_{j} b_{j} F_{y_{j}} .
$$

Now fix a smooth fiber $F$. Then $C_{t} \cdot F=1$ for any $t \in \mathbb{P}^{1}$, so we get a morphism

$$
\mathbb{P}^{1} \longrightarrow F, \quad t \mapsto C_{t} \cap F .
$$

But $F$ is a smooth elliptic curve so this map is constant. We conclude

$$
B \cap F=C_{0} \cap F=C_{\infty} \cap F=B^{\prime} \cap F .
$$

Since $F$ was chosen arbitrary, we deduce that $B=B^{\prime}$ which is a contradiction.

A.2. Weighted Euler characteristics of symmetric products. In this section we prove the following formula for the weighted Euler characteristic of symmetric products. 
LEMMA 32. Let $B$ be a scheme of finite type over $\mathbb{C}$ and let $e(B)$ be its topological Euler characteristic. Let $g: \mathbb{Z}_{\geqslant 0} \rightarrow \mathbb{Z}((p))$ be any function with $g(0)=1$. Let $G: \operatorname{Sym}^{d}(B) \rightarrow \mathbb{Z}((p))$ be the constructible function defined by

$$
G(\boldsymbol{a x})=\prod_{i} g\left(a_{i}\right),
$$

for all $\boldsymbol{a} \boldsymbol{x}=\sum_{i} a_{i} x_{i} \in \operatorname{Sym}^{d}(B)$ where $x_{i} \in B$ are distinct closed points. Then

$$
\sum_{d=0}^{\infty} q^{d} \int_{\operatorname{Sym}^{d}(B)} G d e=\left(\sum_{a=0}^{\infty} g(a) q^{a}\right)^{e(B)} .
$$

REMARK 33. In the special case where $g=G \equiv 1$, the lemma recovers MacDonald's formula

$$
\sum_{d=0}^{\infty} e\left(\operatorname{Sym}^{d}(B)\right) q^{d}=\frac{1}{(1-q)^{e(B)}} .
$$

The lemma is essentially a consequence of the existence of a power structure on the Grothendieck group of varieties defined by symmetric products and the compatibility of the Euler characteristic homomorphism with that power structure. For convenience, we provide a direct proof here.

Proof. The $d$ th symmetric product admits a stratification with strata labeled by partitions of $d$. Associated to any partition of $d$ is a unique tuple $\left(m_{1}, m_{2}, \ldots\right)$ of nonnegative integers with $\sum_{j=1}^{\infty} j m_{j}=d$. The stratum labeled by $\left(m_{1}, m_{2}, \ldots\right)$ parameterizes collections of points where there are $m_{j}$ points of multiplicity $j$. The full stratification is given by

$$
\operatorname{Sym}^{d}(B)=\bigsqcup_{\substack{\left(m_{1}, m_{2}, \ldots\right) \\ \sum_{j=1}^{\infty} j m_{j}=d}}\left\{\left(\prod_{j=1}^{\infty} B^{m_{j}}\right)-\Delta\right\} / \prod_{j=1}^{\infty} \sigma_{m_{j}},
$$

where by convention, $B^{0}$ is a point, $\Delta$ is the large diagonal, and $\sigma_{m}$ is the $m$ th symmetric group. Note that the function $G$ is constant on each stratum and has value $\prod_{j=1}^{\infty} g(j)^{m_{j}}$. Note also that the action of $\prod_{j=1}^{\infty} \sigma_{m_{j}}$ on each stratum is free.

For schemes over $\mathbb{C}$, topological Euler characteristic is additive under stratification and multiplicative under maps which are (topological) fibrations. Thus

$$
\int_{\operatorname{Sym}^{d}(B)} G d e=\sum_{\substack{\left(m_{1}, m_{2}, \ldots\right) \\ \sum_{j=1}^{\infty} j m_{j}=d}}\left(\prod_{j=1}^{\infty} g(j)^{m_{j}}\right) \frac{e\left(B^{\sum_{j} m_{j}}-\Delta\right)}{m_{1} ! m_{2} ! m_{3} ! \ldots} .
$$


For any natural number $N$, the projection $B^{N}-\Delta \rightarrow B^{N-1}-\Delta$ has fibers of the form $B-\{N-1$ points $\}$. The fibers have constant Euler characteristic given by $e(B)-(N-1)$ and consequently, $e\left(B^{N}-\Delta\right)=(e(B)-(N-1)) \cdot e\left(B^{N-1}-\Delta\right)$. Thus by induction, we find $e\left(B^{N}-\Delta\right)=e(B) \cdot(e(B)-1) \cdots(e(B)-(N-1))$ and so

$$
\frac{e\left(B^{\sum_{j} m_{j}}-\Delta\right)}{m_{1} ! m_{2} ! m_{3} ! \cdots}=\left(\begin{array}{c}
e(B) \\
m_{1}, m_{2}, m_{3}, \cdots
\end{array}\right),
$$

where the RHS is the generalized multinomial coefficient.

Putting it together and applying the generalized multinomial theorem, we find

$$
\begin{aligned}
\sum_{d=0}^{\infty} q^{d} \int_{\operatorname{Sym}^{d}(B)} G d e & =\sum_{\left(m_{1}, m_{2}, \ldots\right)} \prod_{j=1}^{\infty}\left(g(j) q^{j}\right)^{m_{j}}\left(\begin{array}{c}
e(B) \\
m_{1}, m_{2}, m_{3}, \ldots
\end{array}\right) \\
& =\left(1+\sum_{j=1}^{\infty} g(j) q^{j}\right)^{e(B)},
\end{aligned}
$$

which proves the lemma.

\section{References}

[1] M. F. Atiyah and I. G. Macdonald, Introduction to commutative algebra (Addison-Wesley Publishing Co., Reading, MA-London-Do Mills, Ont., 1969).

[2] K. Behrend, 'Donaldson-Thomas type invariants via microlocal geometry', Ann. of Math. (2) 170(3) (2009), 1307-1338. arXiv:math/0507523.

[3] K. Behrend and B. Fantechi, 'Symmetric obstruction theories and Hilbert schemes of points on threefolds', Algebra Number Theory 2(3) (2008), 313-345. arXiv:math/0512556.

[4] T. Bridgeland, 'Hall algebras and curve-counting invariants', J. Amer. Math. Soc. 24(4) (2011), 969-998. arXiv:1002.4374.

[5] J. Bryan, 'The Donaldson-Thomas theory of $K 3 \times E$ via the topological vertex', in Geometry of Moduli, (eds. J. A. Christophersen and K. Ranestad) Abel Symposia, 14 (Springer, 2018).

[6] J. Bryan, M. Kool and B. Young, 'Trace identities for the topological vertex', Selecta Math. (N.S.) 24(2) (2018), 1527-1548. arXiv:math/1603.05271.

[7] J. Bryan, G. Oberdieck, R. Pandharipande and Q. Yin, 'Curve counting on abelian surfaces and threefolds', Algebraic Geometry (Compositio) 5(4) (2018), 398-463. arXiv:math/1506.0 0841.

[8] R. Friedman and J. Morgan, Smooth Four-manifolds and Complex Surfaces, Ergebnisse der Mathematik und ihrer Grenzgebiete, 27 (Springer, 1994).

[9] M. Haiman, ' $t, q$-Catalan numbers and the Hilbert scheme', Discrete Math. 193(1-3) (1998), 201-224. Selected papers in honor of Adriano Garsia (Taormina, 1994).

[10] T. Kawai and K. Yoshioka, 'String partition functions and infinite products', Adv. Theor. Math. Phys. 4(2) (2000), 397-485.

[11] M. Kool and R. Thomas, 'Stable pairs with descendents on local surfaces I: the vertical component', Pure Appl. Math. Q. 13(4) (2017), 581-638. arXiv:math/1605.02576. 
[12] R. D. MacPherson, 'Chern classes for singular algebraic varieties', Ann. of Math. (2) 100 (1974), 423-432.

[13] D. Maulik, N. Nekrasov, A. Okounkov and R. Pandharipande, 'Gromov-Witten theory and Donaldson-Thomas theory. I', Compos. Math. 142(5) (2006), 1263-1285. arXiv:math.AG/0 312059.

[14] D. Maulik, R. Pandharipande and R. P. Thomas, 'Curves on $K 3$ surfaces and modular forms', J. Topol. 3(4) (2010), 937-996. With an appendix by A. Pixton.

[15] R. Miranda, The basic theory of elliptic surfaces. Dottorato di Ricerca in Matematica. [Doctorate in Mathematical Research]. ETS Editrice, Pisa, 1989. http://www.math.colostat e.edu/ miranda/BTES-Miranda.pdf.

[16] A. Okounkov, N. Reshetikhin and C. Vafa, 'Quantum Calabi-Yau and classical crystals', in The Unity of Mathematics, Progress in Mathematics, 244 (Birkhäuser Boston, Boston, MA, 2006), 597-618. arXiv:hep-th/0309208.

[17] R. Pandharipande and R. P. Thomas, 'The Katz-Klemm-Vafa conjecture for $K 3$ surfaces', Forum Math. Pi 4(e4) (2016), 111 arXiv:math/1404.6698.

[18] Y. Toda, 'Stability conditions and curve counting invariants on Calabi-Yau 3-folds', Kyoto J. Math. 52(1) (2012), 1-50. arXiv:math/1103.4229. 